\title{
Relación entre la exposición ocupacional a Campos Electromagnéticos y riesgo de desarrollar cáncer: una Revisión Sistemática
}

\section{Relation Between the Occupational Exposure to Electromagnetic Fields and the Risk of Developing Cancer: A Systematic Review}

\section{Sabrina Pozzobon' ', Cristina Rivera Nieto ${ }^{2}$, Ximena Tatiana Lasso Ruales ${ }^{2}$, Ana Bella Alcaraz Martínez' , Alexander Utria Velázquez'}

1. Unidad Docente de Medicina del Trabajo de Madrid. España.

2. Unidad Docente de Medicina del Trabajo de Castilla La Mancha. España.

Recibido: 07-05-2018

Aceptado: 17-05-2019

\section{Correspondencia}

Correo electrónico: criveran@sescam.jccm.es

Este trabajo se ha desarrollado dentro del Programa Científico de la Escuela Nacional de Medicina del Trabajo del Instituto de Salud Carlos III en convenio con la Unidad Docente de Medicina del Trabajo de Castilla la Mancha y la Unidad Docente de Medicina del Trabajo de la Comunidad de Madrid. Madrid. España.

Resumen

Los campos electromagnéticos (CEM) están presentes en todos los ambientes en dónde se utiliza energía eléctrica. Toda la población se encuentra expuesta a CEM, pudiendo éstos producir efectos en la salud.

Este trabajo pretende constatar si existe relación entre la exposición ocupacional a CEM y el desarrollo de cáncer.

Se realizó una búsqueda bibliográfica en siete bases de datos: MEDLINE, COCHRANE, WOK, IBECS, LILACS, EMF-portal y SCOPUS. Como criterio de inclusión, se tuvieron en cuenta los artículos publicados entre 2000 y 2017, el tipo de artículo, el idioma y que la población expuesta a CEM fuese únicamente ocupacional.

La presente revisión sistemática encuentra resultados contradictorios entre la exposición ocupacional a CEM y el desarrollo de cáncer, por tanto, no se puede llegar a una conclusión sólida. La evidencia parece demostrar que altos niveles de exposición a CEM podrían aumentar el riesgo de padecer ciertos tipos de cánceres.

Debido a la diversidad de resultados obtenidos, se deberían realizar nuevos estudios con una mejor calidad metodológica y con un número suficiente de trabajadores expuestos a dosis altas de CEM. Por último, deberían aplicarse en todos los estudios medidas o matrices de exposición homogéneas para permitir la mejor comparación de resultados.

Med Segur Trab (Internet). 2019;65(255):139-59

Palabras clave: campos electromagnéticos, exposición ocupacional, cáncer.

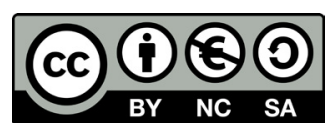

https://creativecommons.org/licenses/by-nc-sa/4.0/ 


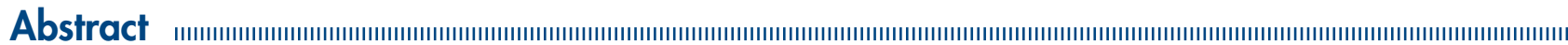

Electromagnetic fields (EMF) are present at any place where electric energy is used. There is a permanent risk of developing adverse health consequences, since population is exposed to EMF.

This work aims to investigate if any relation between the occupational exposure to EMF and its cancer associated risks took place.

A systematic review of the literature was carried out in seven databases: MEDLIE, COCHRANE, WOK, IBECS, LILACS, EMF-portal and SCOPUS. The articles published between 2000 and 2017, the article type, language, and just occupationally exposure population were taken into account in the inclusion criteria.

Contradictory results between occupational EMF exposure and the risk of developing cancer were found by this systematic criteria. Nevertheless, evidence seems to demonstrate that high-level EMF exposure might arise the risk of causing any type of cancer.

Given that diversity of results found, new studies should be done with a better methodological quality and an enough number of workers exposed to EMF high levels. In closing and in order to obtain a better result comparison, standard EMF exposure measurements should in every single future study be applied.

Med Segur Trab (Internet). 2019;65(255):139-59

Keywords: electromagnetic fields, neoplasms, occupational exposure. 


\section{INTRODUCCIÓN}

Los efectos de los campos electromagnéticos han suscitado interés y curiosidad desde la antigüedad. Las primeras observaciones conocidas sobre los fenómenos eléctricos se remontan a Tales de Mileto (624-546 a.C.) ${ }^{1}$.

El electromagnetismo tiene muy diversas manifestaciones: los rayos $\mathrm{X}$, la luz ultravioleta, las ondas de radio y los campos estáticos. Siendo todas emisiones electromagnéticas diferentes, que se pueden caracterizar por su frecuencia, longitud de onda o energía ${ }^{1}$. Según el Real Decreto 299/2016, de 22 de julio de 2016, se entiende por campos electromagnéticos (CEM): los campos eléctricos estáticos, los campos magnéticos estáticos y los campos eléctricos, magnéticos y electromagnéticos variables en el tiempo, con frecuencias comprendidas entre $0 \mathrm{~Hz}$ y $300 \mathrm{GHz}^{2}$. Toda la población se encuentra expuesta en cierto grado a los campos electromagnéticos, por lo tanto, para el estudio de su evaluación es importante separar a las personas más expuestas de las menos expuestas ${ }^{3}$.

Los campos electromagnéticos (CEM) pueden producir efectos sobre la salud. Para ello, es necesario, que exista una interacción entre dichos campos y las estructuras biológicas y que se produzcan en éstas algún cambio fisiológico perceptible o detectable. Estos cambios se denominan "efectos biológicos" y se caracterizan por la existencia de una transferencia energética, que genera una señal que puede ser percibida y amplificada por las células, para producir posteriormente una respuesta del organismo ${ }^{1}$. Por ejemplo, la radiación no ionizante (RNI) que engloba toda la radiación y los campos del espectro electromagnético, no tiene suficiente energía para ionizar la materia y es por tanto incapaz de dañar el $\mathrm{ADN}^{4}$. Esto indica que los CEM no serían agentes mutagénicos "per se" y requerirían la presencia de un precursor para iniciar el desarrollo del cáncer5. Sin embargo, en el año 2002 el Centro Internacional de Investigación sobre el Cáncer (IARC) categorizó las radiaciones electromagnéticas, incluyendo frecuencias hasta $60 \mathrm{hz}$, como posiblemente carcinógeno para los humanos (grupo $2 \mathrm{~B})^{6}$.

En los últimos 44 años se han realizado diversas investigaciones sobre campos electromagnéticos y sus posibles efectos en la salud, publicándose aproximadamente 2900 estudios relacionados con cáncer ${ }^{6}$. Uno de ellos, fue el de la Comisión Internacional de Protección contra la Radiación No Ionizante (ICNIRP, por sus siglas en inglés), que declaró en su revisión que los estudios sobre el riesgo de tumor cerebral y leucemia en adultos en relación con la exposición ocupacional a CEM han variado desde asociaciones nulas a fuertemente positivas ${ }^{7}$. En los estudios realizados sobre cáncer de mama, se plantea la hipótesis de que los CEM suprimen la producción nocturna de melatonina, la cual es un inhibidor de la carcinogénesis mamaria. Por tanto, la reducción de la melatonina aumenta el riesgo de desarrollo de dicho cáncer ${ }^{8}$. Sin embargo, hay otros procesos inducidos por CEM que también aumenta el riesgo de desarrollo de cáncer de mama como: la proliferación de células mamarias cancerígenas, la inhibición del proceso de diferenciación celular y la disrupción de señales de transducción mamaria? .

\section{OBJETIVOS}

El objetivo general de esta revisión sistemática es:

- Constatar si existe relación entre la exposición ocupacional a campos electromagnéticos y el desarrollo de cáncer; ya que los estudios previos son inconsistentes y dicha relación es aún controvertida.

Teniendo como objetivos específicos los siguientes:

- Explorar los diferentes tipos de cánceres clasificándolos en: mama, tumor cerebral, hematológico y otros.

- Valorar la relación de los efectos según el tiempo y nivel de la exposición a CEM de los cánceres más estudiados.

- Observar el nivel de evidencia científica que existe para cada estudio analizado. 


\section{MATERIALES Y MÉTODOS}

Se realizó una búsqueda bibliográfica de artículos científicos, publicados entre 2000 y 2017 en siete bases de datos: MEDLINE, COCHRANE, WOK, IBECS LILACS, EMF-portal, SCOPUS. La última fecha de consulta fue el 23/11/2017. Para dicha búsqueda se utilizaron los descriptores y ecuaciones observados en la tabla I. Posteriormente se procedió a la selección de artículos para su lectura sistemática en base a los criterios de inclusión (tabla II).

Tabla I. Bases de datos. Descriptores y ecuaciones de búsqueda.

\begin{tabular}{l|l}
\hline \multicolumn{1}{c|}{ Bases de datos } & \multicolumn{1}{c}{ Descriptores y ecuaciones } \\
\hline MEDLINE & $\begin{array}{l}(((\text { “neoplasms"[MeSH Terms]) AND (((()ccupational exposure[MeSH Terms]) OR } \\
\text { occupational diseases[MeSH Terms])) AND "electromagnetic fields"[MeSH Major } \\
\text { Topic]) AND Humans[Mesh])) AND ( "2000/01/01"[PDat] : "3000/12/31"[PDat] ) AND } \\
\text { Humans[Mesh] AND adult[MeSH])) OR (((((neoplasm OR cancer OR neoplasia OR } \\
\text { tumor)) AND electromagnetic fields) AND (occupational exposure OR occupational } \\
\text { diseases))) NOT medline [sb]) }\end{array}$ \\
\hline COCHRANE & MeSH descriptor: [Neoplasms] explode all trees \\
\hline & MeSH descriptor: [Occupational Exposure] explode all trees \\
\hline WOK & MeSH descriptor: [Occupational Diseases] explode all trees \\
\hline MeSH descriptor: [Electromagnetic Fields] explode all trees
\end{tabular}

Tabla II. Criterios de inclusión.

\begin{tabular}{l|l}
\hline \multicolumn{1}{c|}{ Variables } & \multicolumn{1}{c}{ Criterios de inclusión } \\
\hline Artículos seleccionados & $\begin{array}{l}\text { Los que relacionan exposición ocupacional a radiaciones electromagnéticas } \\
\text { y cualquier tipo de cáncer }\end{array}$ \\
\hline Tipo & Artículos científicos, revisiones sistemáticas y metaanálisis \\
\hline Población & Trabajadores y trabajadoras \\
\hline Fecha de publicación & A partir del año 2000 \\
\hline Idioma & Español, inglés, francés, italiano y portugués \\
\hline Tipo de exposición & Ocupacional \\
\hline
\end{tabular}

Una vez seleccionados los artículos, se obtienen a texto completo a partir de la biblioteca de la Escuela Nacional de Medicina del Trabajo. Tras la recuperación de los artículos y lectura de éstos, se procede a su valoración siendo rechazados los artículos pertinentes siguiendo los criterios de exclusión (tabla III). Los artículos fueron valorados por cada investigador tras su lectura sistemática y se consensuó en reunión aquellos artículos en los que surgieron dudas para su inclusión.

Para determinar la calidad de la información de cada artículo, se evaluó mediante los criterios STROBE $^{10}$ y PRISMA ${ }^{11}$ de EQUATOR Network, utilizando además estos 
últimos para la elaboración del presente trabajo; y para evaluar la evidencia científica de cada uno, se utilizaron los criterios SIGN de la "Scottish Intercollegiate Guidelines Network"12.

Tabla III. Criterios de exclusión.

\begin{tabular}{l|l}
\hline \multicolumn{1}{c|}{ Variables } & \multicolumn{1}{c}{ Criterios de exclusión } \\
\hline No cumplir criterios de inclusión & \multicolumn{1}{|c}{} \\
\hline Distintas bases de datos & Duplicados o ampliación de estudio previo \\
\hline Población & $<18$ años o hijos de trabajadores expuestos* \\
\hline Resultados & $\begin{array}{l}\text { No distinción entre exposición ocupacional y no ocupacional, } \\
\text { estudios en población general, exposición a telefonía móvil }\end{array}$ \\
\hline Exposición y mortalidad & $\begin{array}{l}\text { Estudios cuya variable principal sea el estudio de la mortalidad, en } \\
\text { lugar del riesgo de padecer cáncer por la exposición a CEM }\end{array}$ \\
\hline Artículos no obtenidos & Tras solicitud previa \\
\hline Estudios no originales & \\
\hline
\end{tabular}

*T. Tynes, T. Haldorsen 2003, T. Tynes et al 2003 y Ulla M.Forssén 2004, incluyen en su análisis estadístico población trabajadora mayor de dieciséis años.

\section{RESULTADOS}

La tabla IV refleja el número total de artículos recuperados en cada una de las bases de datos consultadas tras aplicar las distintas ecuaciones de búsqueda, así como el número de artículos seleccionados para su revisión sistemática de acuerdo al proceso de selección seguido.

Tabla IV. Número de artículos recuperados y seleccionados.

\begin{tabular}{l|c|c|c}
\hline \multicolumn{1}{c|}{ Base de datos } & $\begin{array}{c}\text { Artículos recuperados } \\
\text { sin filtros }\end{array}$ & $\begin{array}{c}\text { Artículos recuperados } \\
\text { con filtros }\end{array}$ & Artículos seleccionados \\
\hline Medline & 230 & 63 & 30 \\
\hline WOK & 3 & 3 & 0 \\
\hline LILACS & 1 & 1 & 0 \\
\hline IBECS & 1 & 0 & 0 \\
\hline COCHRANE & 0 & 0 & 0 \\
\hline SCOPUS & 570 & 22 & 1 \\
\hline EMF-portal & 242 & 62 & 4 \\
\hline Total & 1047 & 151 & 35 \\
\hline
\end{tabular}

Se recuperaron un total de 1047 referencias de las cuales 896 fueron eliminadas por no cumplir los criterios de inclusión o por estar repetidos en las diferentes bases de datos. De los 151 artículos restantes válidos, se procedió a la lectura de los títulos y resúmenes de cada uno y se aplicaron los criterios de exclusión dando como resultado una selección para lectura sistemática un total de 35 artículos para su lectura sistemática (Figura 1).

Desde el punto de vista del análisis metodológico el tipo de diseño de los estudios incluidos en la revisión fueron: 2 metaanálisis, 2 revisiones sistemáticas, 6 estudios de cohortes y 25 estudios de casos-controles.

Villeneuve et al. (2000) ${ }^{13}$ en un estudio casos-control anidado, comparan la exposición a campos eléctricos y electromagnéticos, en sus resultados encuentran asociación entre los campos eléctricos y leucemia, sin lograr los mismos resultados en los campos electromagnéticos > $0.47 \mu \mathrm{T}$ OR $=2.31$ (IC 95\%: 0.66-8.12). 
Figura 1. Diagrama de flujo de los resultados de la búsqueda.

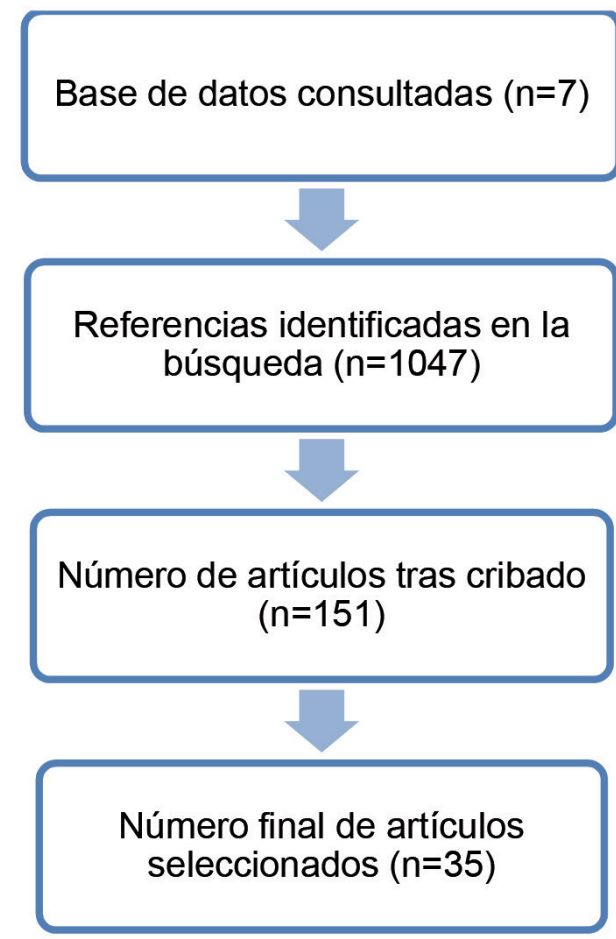

Referencias eliminadas tras aplicar criterios de inclusión $(n=896)$

\section{Artículos eliminados tras aplicar criterios de exclusión $(n=116)$}

Forssén et al. (2000) ${ }^{14}$ realizaron un estudio caso-control en población residente alrededor de 300 metros de líneas de transmisión en Suecia combinado con exposición ocupacional a CEM y su asociación con cáncer de mama femenino. Exposición ocupacional superior a $0.25 \mu \mathrm{T}$ antes del diagnóstico se estima un RR $=1.0$ (IC 95\%: 0.6-1.7). Mujeres menores de 50 años al diagnóstico tienen RR = 1.5 (IC 95\%: 0.6-3.5). Mujeres menores de 50 años y con receptores estrogénicos positivos para cáncer de mama $\mathrm{RR}=3.2$ (IC 95\%: 0.5-18.9). Los resultados fueron similares al combinar exposición residencial y ocupacional.

Fincham et al. $2000^{15}$ realizaron un estudio casos y controles. Toman historias laborales de 1272 pacientes con cáncer y 2666 controles en Canadá, para relacionar el riesgo de algunas profesiones con cáncer de tiroides. Concluyen que la exposición a CEM no afecta al riesgo de padecer este tipo de cáncer OR = 1.59 (IC 95\%: 0.80-3.15).

Van Wijngaarden et al. (2001) ${ }^{16}$ estudian 843 casos de cáncer de mama tomados del Registro de cáncer Central de Carolina del Norte y 773 controles, encontrando que para una exposición acumulada de 20 años o más existían riesgos moderadamente elevados para niveles medios de exposición OR $=1.5$ (IC 95\%: 1.1-2.0), con una asociación más fuerte para mujeres premenopáusicas con exposición en los últimos 10 a 20 años OR = 1.7 (IC 95\%: 1.1-2.7), y aquellas con cáncer de mama y receptor de estrógeno positivo OR $=2.06$ (IC 95\%: 1.1-4.0). Los resultados dan muy poco apoyo a que los CEM tengan una relación dosis-respuesta con cáncer de mama.

Fabbro-Peray et al. (2001) ${ }^{17}$ realizaron un estudio con 445 casos y 1963 controles. Para los radio-operadores, se observó un aumento de riesgo de Linfoma No Hodgkin (LNH) estadísticamente significativo OR $=3.33$ (IC 95\%: 1.5-7.1) p $=0.005$. También para los trabajadores que soldaban diariamente OR $=2.6$ (IC 95\%: 1.4-5.1) $\mathrm{p}=0.005$. Se tuvo en cuenta el tiempo de exposición, encontrándose una asociación estadísticamente significativa para radio-operadores expuestos $\leq 9.5$ años OR $=6.3$ (IC 95\%: 2.1-18.9) $\mathrm{p}=0.001$ y en soldadores expuestos $\leq 13$ años OR $=2.7$ (IC 95\%: 1.3-5.5) p = 0.01. En ambos casos los resultados se encuentran ajustados por edad, género, nivel cultural y localización. Debido al poco número de trabajadores expuestos es necesario confirmar dichos resultados. 
Bethwaite et al. (2001) ${ }^{18}$ estudiaron 110 casos y 199 controles observando un aumento de riesgo de leucemia estadísticamente significativo entre soldadores $\mathrm{OR}=2.8$ (IC 95\%: 1.2-6.8) y trabajadores de línea telefónica OR = 5.81 (IC 95\%:1.2-27.8). Este aumento de riesgo se atribuye a la Leucemia Aguda No Linfocítica (LANL) OR = 2.31 (IC 95\%: 1.2-4.6.) en lugar de a la Leucemia Linfoblástica (LAL).

Pollán et al. (2001) ${ }^{19}$ computaron ratios de incidencia estandarizada de cáncer de mama desde 1971-1989 en una cohorte de hombres suecos con edades entre 25-59 años y lo relacionaron con tipos de ocupación. Encontraron un riesgo relativo en exposiciones por encima del primer cuartil $(0.12 \mu \mathrm{T}) \mathrm{RR}=1.31$ (IC 95\%: 0.94-1.81) sin un claro patrón de exposición-respuesta. No encontraron clara evidencia del rol de los CEM como etiología para desarrollar cáncer de mama en hombres.

Stang et al. (2001) ${ }^{20}$ entrevistaron 118 casos de melanoma uveal y 475 controles, en donde se indagaba por la exposición laboral a CEM y radiación de radiofrecuencia, en este último encontraron asociación con el tumor estudiado, pero con CEM los resultados no fueron concluyentes sobre la fuente de los CEM como máquinas eléctricas OR = 1.0 (IC 95\%: 0.6-1.7) y pantallas de visualización OR = 1.1 (IC 95\%: 0.6-1.9).

Ahlbom et al $(2001)^{8}$ realizó una revisión sistemática que incluye el estudio de la leucemia, tumores cerebrales, cáncer de mama y cáncer de pulmón. De manera general la medición de la exposición ocupacional a CEM es incompleta y difícil. En el caso de la leucemia encontramos desde asociaciones positivas dependientes de dosis respuestas, hasta asociaciones nulas. Respecto a los tumores cerebrales, la asociación fue mayor en aquellos que estudian únicamente gliomas. Sobre el cáncer de mama, son numerosos los estudios que tienen en cuenta los factores de riesgo en la mujer. Pero hay pocos que relacionen a éstas con la exposición ocupacional debido al escaso número de mujeres empleadas en trabajos relacionados con los CEM. Respecto al cáncer de pulmón se encuentra un efecto dosis respuesta en un estudio OR = 6.7 (IC 95\%: 2.7-16.6).

Goodman et al (2002) ${ }^{21}$ hacen una revisión sistemática informando que los factores de confusión (FC) no controlados, ya sean solos o en combinación es poco probable que produzcan una OR espuria de más de 1.5 entre exposición a CEM y cáncer de mama. Sin embargo, los FC descontrolados podrían representar un RR en el rango de 1.2-1.3; y por el contrario una asociación falsa negativa podría ocurrir si el grupo de estudio incluye una gran cantidad de migrantes de Asia y África.

Baumgardt-Elms et al (2002) ${ }^{22}$ realizaron un estudio caso control con 269 casos de cáncer testicular y 797 controles en 5 ciudades alemanas. Para cada una de las 5 categorías de exposición (A-E), se calculó OR estratificada por edad y región. Para la categoría A de exposición ( $44.6 \%$ casos), que incluye trabajar en un complejo ambiente eléctrico, se observó una OR = 0.9 (IC 95\%: 0.67-1.21). Para las otras categorías tampoco se encontró asociación estadísticamente significativa. Por tanto, la exposición a CEM no parece ser factor de riesgo para el cáncer testicular.

Navas-Acién et al. (2002)5 elaboraron un estudio de cohortes, donde analizó la relación entre nivel de exposición a CEM $(<0.13 \mu \mathrm{T}, 0.13-0.20 \mu \mathrm{T},>0.20 \mu \mathrm{T})$ y el riesgo de glioma y meningioma, en hombres suecos trabajadores. Se observó una asociación estadísticamente significativa en el aumento de riesgo de glioma en el segundo y tercer nivel de exposición: [0.13-0.20 $\mu \mathrm{T} ; \mathrm{RR}=1.12$ (IC 95\%: 1.02-1.22)] y [0.20-0.30 $\mu \mathrm{T} ; \mathrm{RR}=1.12$ (IC 95\%: 1.01-1.25)] respectivamente. Sin embargo, no se encontró asociación en los expuestos a $>0.30 \mu \mathrm{T} R \mathrm{RR}=1.07$ (IC 95\%: 0.94-1.91). No se encontró relación entre exposición a CEM y meningioma. Aunque los resultados mencionados deben ser confirmados.

Villeneuve et al. (2002) ${ }^{23}$ realizaron un estudio tipo caso-control con 543 casos y los mismos controles. Se estudió la presencia de astrocitoma, glioblastoma y otros tumores cerebrales en hombres trabajadores de 8 provincias canadienses. No se observó aumento del riesgo de cáncer en hombres con un nivel de exposición $>0.6 \mu \mathrm{T}$ ni $<0.3$ $\mu$ T. Sin embargo, se encontró un aumento estadísticamente significativo en aquellos 
diagnosticados de glioblastoma multiforme OR = 5.36 (IC 95\%: 1.16-24.78), resultado que debe ser interpretado con cautela debido a los pocos diagnósticos de glioblastoma multiforme.

Labrèche et al. (2003) ${ }^{24}$ realizan un estudio con 608 casos y 667 controles en mujeres, diagnosticadas de cáncer de mama entre los 50 y 75 años, expuestas a CEM en su vida laboral. Encuentran asociación estadísticamente significativa en mujeres expuestas a media y alta intensidad $(0.5-10 \mu \mathrm{T})$ antes de los 35 años con tumores con receptor de estrógeno positivo OR = 1.56 (IC 95\%: 1.02-2.39).

Kliukiene et al $(2003)^{25}$ realizan dos estudios en operadoras de radio y telégrafo expuestas a radio frecuencia y frecuencias extremadamente bajas $(50 \mathrm{~Hz})$ : uno tipo cohorte $(n=2619)$ y posteriormente uno tipo caso control anidado (99 casos y 396 controles). Los resultados en el grupo con mayor exposición acumulada fueron $\mathrm{OR}=2.02$ (IC 95\%: 0.97-4.22). Sus resultados sugieren relación entre la dosis acumulada de radiación electromagnética y el riesgo de cáncer de mama.

Willett et al $(2003)^{26}$ realizaron un estudio con 764 casos de trabajadores expuestos a CEM y diagnosticados de leucemia aguda y 1510 controles. Se encontró una relación significativa en el caso de LLA en mujeres expuestas OR = 3.48 (IC 95\%: 1.18-10.2), siendo los demás casos de LLA y LMA no estadísticamente significativos.

Tynes y Haldorsern (2003) ${ }^{27}$ estudiaron 1068 casos diagnosticados de leucemia expuestos a CEM y 2126 controles, en el periodo $x$ años > 31 ( $0.1 \mu \mathrm{T})$. Encontraron asociación no estadísticamente significativa en el aumento de riesgo de leucemia y mieloma por exposición ocupacional a CEM.

Tynes et al (2003) ${ }^{28}$ realizaron un estudio tipo caso control anidado con exposición a CEM (> 0.1 uT) en 807 casos diagnosticados de melanoma y 1614 controles, no encontrando relación estadísticamente significativa en el incremento de riesgo de melanoma por exposición a CEM en el trabajo.

Kliukiene et al $(2004)^{29}$ realizan un estudio de casos y controles analizando 158 casos de cáncer de mama del Registro de Cáncer Noruego y 211 controles. Se observó que las mujeres con la mayor exposición ocupacional tenían una OR = 1.13 (IC 95\%: 0.911.40) en comparación con aquellos que no estaban expuestos en el trabajo, cuyos resultados no mostraron diferencias estadísticamente significativas.

Forssén et al $(2005)^{30}$ estudian en Suecia un total de 20,400 casos de cáncer de mama a partir del Registro Regional de Cáncer de Estocolmo y 116,227 controles seleccionados al azar de la base de estudio. No se encontraron evidencias de un mayor riesgo de cáncer de mama entre las mujeres que trabajan en ocupaciones con alta exposición a CEM. Sin embargo, un mayor riesgo de cáncer de mama podría estar en relación con algunas exposiciones que no sean los CEM.

Klaeboe et al $(2005)^{31}$ realizaron un estudio caso control anidado que midió el riesgo de tumor cerebral teniendo en cuenta la exposición ocupacional y residencial a CEM, analizándose por separado. Los resultados para la exposición ocupacional mostraron una asociación inversa.

Berg et al (2006) ${ }^{32}$ realizaron un estudio caso control con 366 gliomas, 381 meningiomas y 1494 controles. No se encontraron asociaciones estadísticamente significativas entre el riesgo de dichos cánceres y la exposición ocupacional a campos electromagnéticos de radiofrecuencia / microondas.

Lope et al. (2006) ${ }^{33}$ elaboraron un estudio de cohortes histórico de 2.992 .166 trabajadores hombres y mujeres de Suecia seguidos entre 1971 y 1989. Se midió la exposición ocupacional a CEM a través de 3 matrices de exposición laboral. Obtuvieron como resultado que la exposición ocupacional a CEM no presentó efectos sobre el riesgo de padecer cáncer de tiroides. Sin embargo, las mujeres expuestas a altas dosis de radiación si presentaron un aumento del riesgo $\mathrm{RR}=1.85$ (IC 95\%: 1.02-3.35). 
McElroy et al. (2007) ${ }^{9}$ realizaron un estudio de casos y controles en mujeres que durante su vida laboral habían estado expuestas a CEM, siendo 6213 casos y 7390 controles. Sus resultados sugieren que la exposición a altas dosis de CEM puede estar asociadas con una ligera elevación del riesgo de cáncer de mama (para exposición alta OR = 1.16 (IC 95\%: 0.9-1.5)), para exposición media OR = 1.09 (IC 95\%: 0.96-1.23) y para baja exposición OR = 1.06 (IC 95\%: 0.99-1.14).

Johansen et al. (2007) ${ }^{34}$ estudian los riesgos para leucemia, cáncer cerebral y cáncer de mama en una cohorte de empleados de servicios públicos y privados que suministran electricidad en Dinamarca. No se encontró un aumento en el riesgo de estos cánceres entre 28.224 sujetos con más de 3 meses de empleo, en quienes no se había diagnosticado cáncer antes del primer empleo.

Karipidis et al. (2007) $)^{35}$ realizaron un estudio de casos y controles con 414 casos histológicamente confirmados de glioma (diferenciados en alto y bajo grado) y 412 controles de Melbourne, Australia. Recolectaron la historia ocupacional detallada y estimaron la exposición ocupacional a CEM a través de 3 métodos: auto-reportado, revisión por un higienista experto y a través de una matriz de exposición laboral (JEM). Obtuvieron resultados estadísticamente más elevados, pero no significativos cuando la exposición fue valorada por un higienista experto. Encontraron en el grupo con mayor exposición OR = 1.4 (IC 95\%: 0.85-2.27) para todos los gliomas y OR = 1.51 (IC 95\%: 0.902.53) para gliomas de alto grado.

Karipidis et al. $(2007)^{36}$ realizaron un estudio de casos y controles, (416 casos y 422 controles) considerando como casos aquellos gliomas histológicamente confirmados, diagnosticados entre 1987 y 1991 en Melbourne Australia, y una historia ocupacional detallada. La exposición ocupacional a CEM se midió a través de la matriz de exposición laboral finlandesa (FINJEM). En expuestos de 0-0.75 $\mu \mathrm{T}$ obtuvieron una OR $=0.76$ (IC 95\%: 0.52-1.11), en expuestos de 0,75-2,5 $\mu \mathrm{T}$ OR $=0,80$ (IC 95\%: 0.55-1.17) y en expuestos a $>2,5 \mu \mathrm{T}$ con una $\mathrm{p}=0.20 \mathrm{OR}=0.79$ (IC 95\%: 0.58-1.18). Concluyendo que no encontrados evidencia de que exista relación entre la aparición de glioma y la exposición ocupacional a CEM.

Karipidis et al. (2007) ${ }^{37}$ realizaron un estudio de casos y controles con 694 casos de LNH y 694 controles de 2 regiones de Australia, tomando la historia ocupacional de cada uno y midieron la exposición ocupacional a campos electromagnéticos a través de una matriz de exposición laboral realizada en EEUU. Obtuvieron un valor total de OR $=1.48$ (IC 95\%: 1.02-2.16) en los niveles de mayor exposición y concluyeron que sus resultados apoyan levemente la hipótesis del aumento de riesgo de LNH antes la exposición de CEM.

Kheifets et al. (2008) ${ }^{38}$ realizaron una actualización de 2 metaanálisis que evalúan la exposición ocupacional a campos electromagnéticos con tumores cerebrales y leucemia. Obtuvieron $\mathrm{OR}=1.14$ (IC 95\%: 1.07-1.22) para 47 estudios de tumores cerebrales $\mathrm{y}$ OR $=1.16$ (IC 95\%: 1.11-1.22) para 56 estudios de todos los tipos de leucemia, evidenciando para ambos tipos de cáncer un ligero aumento del riesgo de 10\% y 13\% respectivamente en relación a la exposición ocupacional a CEM.

Coble et al. (2009) ${ }^{39}$ realizaron un estudio de casos y controles en 3 hospitales de EEUU. Se obtuvieron 489 casos de glioma, 197 casos de meningioma y 799 controles. La historia ocupacional se obtuvo a través de cuestionarios y la exposición ocupacional evaluada a través de la matriz de exposición laboral (JEM) con modificaciones basadas en el puesto de trabajo. Obtuvieron como resultado para gliomas una OR $=0.8$ (IC 95\%: 0.61.1) en expuestos entre 1,5-3.0 $\mathrm{mG}$ y OR $=0.8$ (IC 95\%: 0.6-1.1) en expuestos a $>3.0 \mathrm{mG}$, con una $\mathrm{p}=0,19$. En el caso de meningiomas obtuvieron una OR $=1.0$ (IC 95\%: 0.7-1.6) en expuestos entre 1,5-3.0 mG y OR = 1.0 (IC 95\%: 0.6-1.8) en expuestos a $>3.0 \mathrm{mG}$, con una $\mathrm{p}=0.79$. Concluyendo que no existe relación entre la exposición ocupacional a CEM y el aumento del riesgo de padecer gliomas o meningiomas.

Behrens et al $(2010)^{40}$ realizan un estudio con 293 pacientes con melanoma uveal y 3198 sujetos control en 9 países europeos, encontrando que las mujeres expuestas a 
instalaciones de transmisión eléctrica mostraron riesgos elevados OR = 5,81 (IC 95\%: 1.72-19.66). Se observaron asociaciones positivas con la exposición a CEM en salas de control entre hombres y mujeres, pero la mayoría de los aumentos de riesgo se restringieron a sujetos con color de iris oscuro, con un mayor aumento del riesgo entre las mujeres en comparación con los hombres, concluyendo que las mujeres expuestas de ojos oscuros pueden estar en particular riesgo de padecer melanoma uveal.

Baldi et al. (2011) ${ }^{41}$ estudiaron en el suroeste de Francia entre mayo de 1999 y abril de 2000 un total de 221 tumores del sistema nervioso central: 105 gliomas, 67 meningiomas, 33 neurinomas y 16 de otras estirpes, y 442 controles emparejados por sexo seleccionados de la población general. Se encontró un aumento no significativo en el riesgo de exposición ocupacional a CEM OR $=1.52$ (IC 95\%: 0.92-2.51), este aumento se volvió significativo para los meningiomas, especialmente cuando se consideró CEM por separado $\mathrm{OR}=3.02$ (IC 95\%: 1.10-8.25). Estos datos sugieren que la exposición ocupacional o residencial a CEM puede jugar un papel en la aparición de meningioma.

Sun et al. (2013) ${ }^{42}$ realiza un metaanálisis con 7 estudios de casos y controles y 11 estudios de cohortes sobre la exposición a campos electromagnéticos y el riesgo de cáncer de mama masculino. Se encontró un aumento estadísticamente significativo OR agrupadas $=1.32$ (IC 95\%: 1.14-1.52) $\mathrm{p}<0.001, \mathrm{y}$ los análisis de subgrupos también arrojaron resultados similares, sugiriendo que la exposición a los CEM puede asociarse con el aumento del riesgo de cáncer de mama masculino.

Koeman et al. (2014) ${ }^{43}$ elaboraron un estudio de cohorte prospectivo en Países Bajos con 120,852 entre hombres y mujeres, encontrando para la exposición elevada asociación significativa para LMA y LF y para exposición acumulativa asociación positiva y significativa con LF, pero no con LMA entre hombres. No evidencian asociación para cáncer de pulmón, mama ni cerebro.

Carlberg et al (2017)44 estudiaron 1346 casos de glioma y 3485 controles en Suecia encontrando un aumento del riesgo del astrocitoma grado IV (glioblastoma multiforme) para la exposición ocupacional a CEM. La exposición acumulada para el glioblastoma multiforme dio para las categorías de exposición más alta en la ventana de tiempo 1-14 años una OR $=1.9$ (IC 95\%: 1.4-2.6) $p<0,001$, y en la ventana de tiempo de 15 años o más una $\mathrm{OR}=0.9$ (IC 95\%: 0.6-1.3) $p=0.44$.

\section{DISCUSIÓN Y CONCLUSIONES}

Los CEM a diferencia de otros agentes ambientales, están presentes en todos los espacios donde se utiliza energía eléctrica, por lo que los potenciales efectos de exposiciones no ocupacionales a CEM no fueron considerados en la mayoría de los estudios analizados.

Encontramos resultados contradictorios sobre la posibilidad que exista una relación causal entre CEM de baja frecuencia y tumores, ya que varios de los cánceres estudiados son de origen multifactorial. Los estudios analizados sugieren que los CEM pueden ser una de sus causas, sin lograr obtener datos consistentes que lo justifiquen. El periodo de exposición para el desarrollo del cáncer es previo a la fecha en las que se realizan las mediciones. Por tanto, se desconoce el tiempo de inducción exacto para la aparición de esta enfermedad ${ }^{45}$.

Dentro de las limitaciones de nuestra revisión sistemática, encontramos diferentes métodos y unidades para la medición de los niveles de exposición a CEM; así como diversidad de variables, población estudiada y tiempo de seguimiento. Algunos estudios utilizan matrices, tablas y opiniones de expertos; es decir no hay un protocolo estándar para su cuantificación. Por ejemplo, las estimaciones de exposición a CEM para una misma ocupación, obtenidas a través de matrices de exposición laboral, pueden no ser precisas debido a las diferentes condiciones que tienen los trabajadores en función del 
país en el que se aplica. Si tenemos en cuenta la fecha de recolección de datos observamos que en la mayoría de los estudios es previa al año 2000, por lo que se deben tener en cuenta los avances tecnológicos durante los últimos años; ya que actualmente es prácticamente imposible evitar la exposición a las radiaciones electromagnéticas. El gran desarrollo experimentado en los últimos años por la tecnología de los sistemas de comunicación ha provocado el aumento de aplicaciones en radio, televisión, satélites, líneas telefónicas, radares y antenas de telecomunicaciones ${ }^{46}$.

Otra de las limitaciones, es que la mayoría de los estudios son retrospectivos y pueden aparecer sesgos de memoria en aquellos en los que se aplican encuestas a los participantes sobre su historia ocupacional. En los estudios de casos y controles habría que tener en cuenta el sesgo de selección, ya que en algunos los datos fueron tomados de registros electorales o censos poblaciones dando lugar a heterogeneidad dentro de los grupos y dificultando por ello el análisis de los datos. Por otro lado, el periodo de latencia del cáncer es muy largo, lo que dificulta la determinación de la asociación de exposición a CEM con la aparición de la enfermedad.

La principal fortaleza de nuestro estudio es que se trata de una revisión sistemática de la literatura científica desde el año 2000 hasta la actualidad; lo que se traduce en un amplio período de observación en comparación con lo realizado previamente. Además, se ha revisado una amplia cantidad de estudios que abordan la posible relación de los CEM con varios tipos de cáncer; centrándose la mayoría de los estudios previos en el análisis de un solo tipo de ellos. Otra fortaleza de nuestra revisión es que realizamos el análisis por tiempo y niveles de exposición, estos parámetros nos ayudan a hacernos una mejor idea de la exposición ocupacional a CEM; ya que la mayoría de los estudios, generalmente se centran sólo en uno de los dos aspectos. Por último, debemos destacar que se ha tenido en cuenta el nivel de evidencia científico de cada uno de los artículos, obteniendo como mínimo 2- según los criterios SIGN; hecho sin duda diferencial y que aporta calidad a la revisión.

Dentro de los 9 artículos que estudian la relación entre CEM y tumores hematológicos, de éstos 3 no encuentran resultados significativos ${ }^{13,28,34}$ y sólo 6 encontraron asociación estadísticamente significativa para al menos un tipo de cáncer: Willett et al. ${ }^{26}$ encuentran relación entre LLA y mujeres, Koeman et al. ${ }^{43}$ obtuvieron resultados significativos para LMA y LF en hombres con exposición elevada y además LF en exposición acumulada, mientras que Karipidis et al. ${ }^{37}$ encuentran relación entre $\mathrm{LNH}$ y niveles altos de exposición sin distinción de género. Fabbro-Peray et al. ${ }^{17}$ estudiaron la exposición en ocupaciones evidenciando relaciones estadísticamente significativas para LNH y CEM en radio-operadores y soldadores. Por su parte Bethwaite et al.18 encuentran la misma relación con leucemia aguda en los mismos trabajadores. En un metaanálisis realizado por Kheifets et al. ${ }^{38}$ de 47 artículos, encuentra un ligero aumento del riesgo de padecer leucemia. En general se encuentran resultados contradictorios, siendo las asociaciones significativas únicamente en algunos estudios y en grupos de trabajadores con los niveles de exposición a CEM más altos.

De los once estudios que abordaron la posible relación entre la exposición a CEM y tumores cerebrales seis de ellos encuentran una asociación significativa con dicho factor de riesgo para al menos un tipo de cáncer, ${ }^{5,8,23,38,41,44}$. De los cuales, uno de ellos, Navas et al. ${ }^{5}$ muestra en las categorías de exposición más altas un aumento del riesgo para los gliomas. Para Villeneuve et al. ${ }^{23}$ dicha asociación se estableció para la categoría de exposición más alta, sólo con el glioblastoma multiforme, cuyo resultado es similar al de Calberg et al. ${ }^{44}$, el cual tiene en cuenta los años de exposición. Y Kheifehts et al. ${ }^{38}$ que es un metaanálisis obtiene un resultado significativo en 47 de sus estudios. Los dos restantes $^{8,41}$, muestran solo asociación para glioma y meningioma respectivamente. El resto de los estudios sobre los tumores cerebrales, no mostraron asociación con la exposición a CEM para ninguna de las variantes histopatológicas ni para ninguno de los diferentes niveles de exposición estudiados ${ }^{31,32,35,36,39}$.

En relación a la exposición a CEM y su posible papel como factor de riesgo para el cáncer de mama encontramos en total 11 artículos, de los cuales, 5 tuvieron resultados 
estadísticamente no significativos ${ }^{14,29,30,34,43}$, otros 6 con resultados dispares o significativos para un grupo concreto del estudio: Pollán et al. ${ }^{19}$ obtienen significación en exposiciones medias, sin claro patrón exposición-respuesta; McElroy et al. ${ }^{9}$ y Kliuliene et al. ${ }^{25}$ sugieren en sus resultados que hay relación entre la exposición a CEM y la aparición de cáncer de mama cuando se alcanzan dosis de exposición o dosis de exposición acumulada altas; por otro lado, Van Wijngaarden et al. ${ }^{16} \mathrm{y}$ Labrèche et al. ${ }^{24}$ obtienen resultados no significativos, excepto para el grupo de mujeres expuestas a CEM de media-alta intensidad o alta dosis, especialmente si la exposición es anterior a los 35 años en el ámbito laboral y desarrollaron cáncer de mama receptor estrogénico positivo, en cuyo caso sí encuentran relación estadísticamente significativa. Por último, destacamos el metaanálisis realizado por Sun et al. ${ }^{42}$ donde se encontró relación estadísticamente significativa, con alto nivel de evidencia, para la exposición a CEM en medio laboral y el desarrollo posterior de cáncer de mama en hombres.

Entre todos los cánceres estudiados en relación a los CEM, la mayoría de los estudios se centran en cáncer de mama, tumores cerebrales y hematológicos. Sin embargo, se encontraron otros estudios que analizaban la relación entre CEM y cáncer de tiroides ${ }^{15,33}$, testicular ${ }^{47}$, melanoma ${ }^{28}$, melanoma uveal ${ }^{20,40}$ y cáncer de pulmón ${ }^{43}$. No se encontraron asociaciones estadísticamente significativas en el cáncer testicular ni en melanoma. Sin embargo, las mujeres con alta exposición presentaron un ligero aumento de riesgo de cáncer de tiroides $^{33}$ y de melanoma uveal ${ }^{40}$. Mientras que los hombres altamente expuestos $^{43}$ tenían un mayor riesgo de cáncer de pulmón.

Tras la revisión de la literatura científica, no se puede llegar a ninguna conclusión sólida sobre la asociación de exposición ocupacional a CEM y el riesgo de aparición de cáncer. Sin embargo, hay evidencias débiles de que altos niveles de exposición podrían aumentar el riesgo de padecer ciertos tipos de cáncer. Debido a que no existe un patrón definido y homogéneo para la medición de exposición a CEM, la comparación entre los distintos estudios es difícil de analizar. Por lo tanto, el objetivo principal de futuros estudios debería ser: realizar nuevas investigaciones con una mejor calidad metodológica y con un número suficiente de trabajadores con exposición elevada a CEM, basadas en medidas de exposición unificadas ${ }^{45}$.

\section{GLOSARIO}

- $\mu$ T: micro Tesla

- CEM: Campos electromagnéticos

- EE.UU: Estados Unidos

- ELF: Electric fields

- EMF: electromagnetic fields

- FC: factores de confusión

- FINJEM: Finnish Job Exposure Matrix

- HR: Hazard ratio

- Hz: Hercios

- IC: Intervalo de confianza

- JEM: Job Exposure Matrix

- LA: Leucemia aguda

- LAL: Leucemia aguda linfoblástica

- LANL: Leucemia aguda no linfoblástica

- LF: Linfoma folicular

- LMA: Leucemia mieloide aguda

- LNH: Linfoma no Hogdkin 
- mG: miligray

- OR: Odds ratio

- RF-MW EMF: Radiofrequency microwave electromagnetic fields

- RR: Riesgo relativo

- SIGN: Scottish Intercollegiate Guidelines Network

- SOC: Standard Occupational Classification

- TWA: Time-weighted average

\section{REFERENCIAS BIBLIOGRÁFICAS}

1. Panadero G, Rupérez Calvo M-J. La exposición laboral a campos eléctricos y magnéticos estáticos. $1^{a}$ ed. Madrid: Instituto Nacional de Seguridad y Salud en el Trabajo; 2004. [Internet]. [citado 16 de enero de 2018]. Disponible en: https://www.insst.es/documents/94886/96076/campo+electrico/d25478c1-324940cb-9bfe-0880a97c5171

2. Real Decreto $299 / 2016$, de 22 de julio, sobre la protección de la salud y la seguridad de los trabajadores contra los riesgos relacionados con la exposición a campos electromagnéticos. BOE $n^{\circ} 182$ 29-07-2016. [Internet]. [citado 18 de enero de 2018]. Disponible en: https://www.boe.es/boe_gallego/dias/2016/07/29/ pdfs/BOE-A-2016-7303-G.pdf

3. International Agency for Research on Cancer Working Group on the Evaluation of Carcinogenic Risks to Humans. Non-Ionizing Radiation, Part 1: Static and Extremely Low-Frequency (ELF) Electric and Magnetic Fields. Vol. 80. Lyon: IARCPress; 2002. 429 p. [Internet]. [citado 16 de enero de 2018]. Disponible en: https://www.ncbi.nlm.nih.gov/books/NBK390731/pdf/Bookshelf_NBK390731.pdf

4. Knave B. Radiaciones No Ionizantes. En: Mager J, director. Enciclopedia de Seguridad y Salud en el Trabajo. 1 ed. Madrid: Ministerio de Trabajo, Migraciones y Seguridad Social; 1998. p. 49.1-49.35. [Internet]. [citado 19 de enero de 2018]. Disponible en: https://www.insst.es/documents/94886/162520/ Cap\%C3\%ADtulo+49.+Radiaciones+no+ionizantes

5. Navas-Acién A, Pollán M, Gustavsson P, Floderus B, Plato N, Dosemeci M. Interactive effect of chemical substances and occupational electromagnetic field exposure on the risk of gliomas and meningiomas in Swedish men. Cancer Epidemiol Biomarkers Prev. [Internet]. Diciembre 2002 [citado 19 de enero de 2018]; 11(12):1678-83. Disponible en: http://cebp.aacrjournals.org/content/11/12/1678.long

6. Repacholi M. Concern that "EMF" magnetic fields from power lines cause cancer. Sci Total Environ [Internet]. 1 de junio de 2012 [citado 20 de enero de 2018]; 426:454-8. Disponible en: https://www. sciencedirect.com/science/article/pii/S0048969712003919?via\%3Dihub

7. Röösli M, Lörtscher M, Egger M, Pfluger D, Schreier N, Lörtscher E, et al. Leukaemia, brain tumours and exposure to extremely low frequency magnetic fields: cohort study of Swiss railway employees. Occup Environ Med. [Internet]. Agosto de 2007 [citado 20 enero de 2018]; 64(8):553-9. Disponible en: https:// www.ncbi.nlm.nih.gov/pmc/articles/PMC2078497/pdf/553.pdf

8. Ahlbom IC, Cardis E, Green A, Linet M, Savitz D, Swerdlow A, et al. Review of the epidemiologic literature on EMF and Health. Environ Health Perspect. [Internet]. Diciembre de 2001 [citado 20 enero de 2018]; 109 Supl6:911-33. Disponible en: https://www.ncbi.nlm.nih.gov/pmc/articles/PMC1240626/pdf/ehp109s-000911. pdf

9. McElroy JA, Egan KM, Titus-Ernstoff L, Anderson HA, Trentham-Dietz A, Hampton JM, et al. Occupational exposure to electromagnetic field and breast cancer risk in a large, population-based, case-control study in the United States. J Occup Environ Med. [Internet]. Marzo de 2007 [citado 20 enero de 2018]; 49(3):26674. Disponible en: https://insights.ovid.com/pubmed?pmid=17351512

10. Elm E von, Altman DG, Egger M, Pocock SJ, Gøtzsche PC, Vandenbroucke JP. The Strengthening the Reporting of Observational Studies in Epidemiology (STROBE) Statement: Guidelines for Reporting Observational Studies. Epidemiology [Internet]. 1 de noviembre de 2007 [citado 31 de enero de 2018]; 18(6):800-4. Disponible en: https://www.jclinepi.com/article/S0895-4356(07)00436-2/pdf

11. Moher D, Liberati A, Tetzlaff J, Altman DG, and the PRISMA Group. Preferred Reporting Items for Systematic Reviews and Meta-Analyses: The PRISMA Statement. Ann Intern Med. [Internet]. 18 de agosto 2009 [citado 13 de enero de 2018]; 151:264-269. Disponible en: http://annals.org/aim/fullarticle/744664/ preferred-reporting-items-systematic-reviews-meta-analyses-prisma-statement?resultClick=3

12. Harbour R, Miller J. A new system for grading recommendations in evidence based guidelines. BMJ [Internet]. 11 de agosto de 2001 [citado 31 de enero de 2018]; 323(7308):334-6. Disponible en: https:// www.ncbi.nlm.nih.gov/pmc/articles/PMC1120936/pdf/334.pdf 
13. Villeneuve PJ, Agnew DA, Miller AB, Corey PN, Purdham JT. Leukemia in electric utility workers: the evaluation of alternative indices of exposure to $60 \mathrm{~Hz}$ electric and magnetic fields. Am J Ind Med [Interntet]. Junio de 2000 [citado 31 de enero de 2018]; 37(6):607-17. Disponible en: https://www. researchgate.net/publication/229926294_Leukemia_in_electric_utility_workers_The_evaluation_of_ alternative_indices_of_exposure_to_60_Hz_electric_and_magnetic_fields

14. Forssén UM, Feychting M, Rutqvist LE, Floderus B, Ahlbom A. Occupational and residential magnetic field exposure and breast cancer in females. Epidemiology. [Internet]. Enero de 2000 [citado 31 de enero de 2018]; 11(1):24-9. Disponible en: https://insights.ovid.com/pubmed?pmid=10615839

15. Fincham SM, Ugnat AM, Hill GB, Kreiger N, Mao Y. Is occupation a risk factor for thyroid cancer? Canadian Cancer Registries Epidemiology Research Group. J Occup Environ Med. [Internet]. Marzo de 2000 [citado 31 de enero de 2018]; 42(3):318-22. Disponible en: https://insights.ovid.com/ pubmed?pmid=10738710

16. Van Wijngaarden E, Nylander-French LA, Millikan RC, Savitz DA, Loomis D. Population-based casecontrol study of occupational exposure to electromagnetic fields and breast cancer. Ann Epidemiol. [Internet]. Julio de 2001 [citado 1 de febrero de 2018]; 11(5):297-303. Disponible en: https://insights.ovid. com/pubmed?pmid=10738710

17. Fabbro-Peray P, Daures JP, Rossi JF. Environmental risk factors for non-Hodgkin's lymphoma: a population-based case-control study in Languedoc-Roussillon, France. Cancer Causes Control. [Internet]. Abril de 2001 [citado 1 de febrero de 2018]; 12(3):201-12. Disponible en: https://www.ncbi.nlm.nih.gov/ pubmed/11405325

18. Bethwaite P, Cook A, Kennedy J, Pearce N. Acute leukemia in electrical workers: a New Zealand casecontrol study. Cancer Causes Control. [Internet]. Octubre de 2001 [citado 1 de febrero de 2018]; 12(8):6839. Disponible en: https://www.ncbi.nlm.nih.gov/pubmed/11562108

19. Pollán M, Gustavsson P, Floderus B. Breast cancer, occupation, and exposure to electromagnetic fields among Swedish men. Am J Ind Med. [Internet]. Marzo de 2001 [citado 4 de febrero de 2018] ;39(3):276-85. Disponible en: https://www.ncbi.nlm.nih.gov/pubmed/11241560

20. Stang A, Anastassiou G, Ahrens W, Bromen K, Bornfeld N, Jöckel KH. The possible role of radiofrequency radiation in the development of uveal melanoma. Epidemiology. [Internet]. Enero de 2001 [citado 4 de febrero de 2018]; 12(1):7-12. Disponible en: https://insights.ovid.com/pubmed?pmid=11138823

21. Goodman M, Kelsh M, Ebi K, Iannuzzi J, Langholz B. Evaluation of potential confounders in planning a study of occupational magnetic field exposure and female breast cancer. Epidemiology. [Internet]. Enero de 2002 [citado 4 de febrero de 2018]; 13(1):50-8. Disponible en: https://insights.ovid.com/ pubmed?pmid=11805586

22. Baumgardt-Elms C, Ahrens W, Bromen K, Boikat U, Stang A, Jahn I, et al. Testicular cancer and electromagnetic fields (EMF) in the workplace: results of a population-based case-control study in Germany. Cancer Causes Control. [Internet]. Diciembre de 2002 [citado 4 de febrero de 2018]; 13(10):895902. Disponible en: https://www.ncbi.nlm.nih.gov/pubmed/12588085

23. Villeneuve PJ, Agnew DA, Johnson KC, Mao Y, Canadian Cancer Registries Epidemiology Research Group. Brain cancer and occupational exposure to magnetic fields among men: results from a Canadian population-based case-control study. Int J Epidemiol. [Internet]. Febrero de 2002 [citado 4 de febrero de 2018]; 31(1):210-7. Disponible en: https://www.ncbi.nlm.nih.gov/pubmed/11914323

24. Labrèche F, Goldberg MS, Valois M-F, Nadon L, Richardson L, Lakhani R, et al. Occupational exposures to extremely low frequency magnetic fields and postmenopausal breast cancer. Am J Ind Med. [Internet]. Diciembre de 2003 [citado 6 de febrero de 2018]; 44(6):643-52. Disponible en: https://onlinelibrary.wiley. com/doi/abs/10.1002/ajim.10264

25. Kliukiene J, Tynes T, Andersen A. Follow-up of radio and telegraph operators with exposure to electromagnetic fields and risk of breast cancer. Eur J Cancer Prev. [Internet]. Agosto de 2003 [citado 6 de febrero de 2018]; 12(4):301-7. Disponible en: https://insights.ovid.com/pubmed?pmid=12883383

26. Willett EV, McKinney PA, Fear NT, Cartwright RA, Roman E. Occupational exposure to electromagnetic fields and acute leukaemia: analysis of a case-control study. Occup Environ Med. 2003; 60(8): $577-83$

27. Tynes T, Haldorsen T. Residential and occupational exposure to $50 \mathrm{~Hz}$ magnetic fields and hematological cancers in Norway. Cancer Causes Control [Internet]. Octubre de 2003 [citado 8 de enero de 2018]; 14(8):715-20. Disponible en: https://link.springer.com/article/10.1023/A:1026331807952

28. Tynes T, Klaeboe L, Haldorsen T. Residential and occupational exposure to $50 \mathrm{~Hz}$ magnetic fields and malignant melanoma: a population based study. Occup Environ Med. 2003; 60(5): 343-7.

29. Kliukiene J, Tynes T, Andersen A. Residential and occupational exposures to 50-Hz magnetic fields and breast cancer in women: a population-based study. Am J Epidemiol. 2004; 159(9): 852-61.

30. Forssén UM, Rutqvist LE, Ahlbom A, Feychting M. Occupational magnetic fields and female breast cancer: a case-control study using Swedish population registers and new exposure data. Am J Epidemiol. 2005; 161(3): 250-9. 
31. Klaeboe L, Blaasaas KG, Haldorsen T, Tynes T. Residential and occupational exposure to 50-Hz magnetic fields and brain tumours in Norway: a population-based study. Int J Cancer. 2005; 115(1): 137-41.

32. Berg G, Spallek J, Schüz J, Schlehofer B, Böhler E, Schlaefer K, et al. Occupational exposure to radio frequency/microwave radiation and the risk of brain tumors: Interphone Study Group, Germany. Am J Epidemiol. 2006; 164(6): 538-48.

33. Lope V, Pérez-Gómez B, Aragonés N, López-Abente G, Gustavsson P, Floderus B, et al. Occupational exposure to ionizing radiation and electromagnetic fields in relation to the risk of thyroid cancer in Sweden. Scand J Work Environ Health. 2006; 32(4): 276-84.

34. Johansen C, Raaschou O, Olsen JH, Schüz J. Risk for leukaemia and brain and breast cancer among Danish utility workers: a second follow-up. Occup Environ Med. 2007; 64(11): 782-4.

35. Karipidis KK, Benke G, Sim MR, Yost M, Giles G. Occupational exposure to low frequency magnetic fields and the risk of low grade and high grade glioma. Cancer Causes Control. 2007; 18(3): 305-13.

36. Karipidis KK, Benke G, Sim MR, Kauppinen T, Giles G. Occupational exposure to ionizing and nonionizing radiation and risk of glioma. Occup Med (Lond). 2007; 57(7): 518-24.

37. Karipidis K, Benke G, Sim M, Fritschi L, Yost M, Armstrong B, et al. Occupational exposure to power frequency magnetic fields and risk of non-Hodgkin lymphoma. Occup Environ Med [Internet]. Enero de 2007 [citado 2 de enero de 2018]; 64(1):25-9. Disponible en: https://www.ncbi.nlm.nih.gov/pmc/articles/ PMC2092592/

38. Kheifets L, Monroe J, Vergara X, Mezei G, Afifi AA. Occupational electromagnetic fields and leukemia and brain cancer: an update to two meta-analyses. J Occup Environ Med. 2008; 50(6): 677-88.

39. Coble JB, Dosemeci M, Stewart PA, Blair A, Bowman J, Fine HA, et al. Occupational exposure to magnetic fields and the risk of brain tumors. Neuro-Oncol [Internet]. Junio de 2009 [citado 2 de enero de 2018]; 11(3):242-9. Disponible en: https://www.ncbi.nlm.nih.gov/pmc/articles/PMC2718968/

40. Behrens T, Lynge E, Cree I, Sabroe S, Lutz J-M, Afonso N, et al. Occupational exposure to electromagnetic fields and sex-differential risk of uveal melanoma. Occup Environ Med. 2010; 67(11): 751-9.

41. Baldi I, Coureau G, Jaffré A, Gruber A, Ducamp S, Provost D, et al. Occupational and residential exposure to electromagnetic fields and risk of brain tumors in adults: a case-control study in Gironde, France. Int J Cancer. 2011; 129(6): 1477-84.

42. Sun J-W, Li X-R, Gao H-Y, Yin J-Y, Qin Q, Nie S-F, et al. Electromagnetic field exposure and male breast cancer risk: a meta-analysis of 18 studies. Asian Pac J Cancer Prev. 2013; 14(1): 523-8.

43. Koeman T, van den Brandt PA, Slottje P, Schouten LJ, Goldbohm RA, Kromhout H, et al. Occupational extremely low-frequency magnetic field exposure and selected cancer outcomes in a prospective Dutch cohort. Cancer Causes Control. 2014; 25(2): 203-14.

44. Carlberg M, Koppel T, Ahonen M, Hardell L. Case-control study on occupational exposure to extremely low-frequency electromagnetic fields and glioma risk. Am J Ind Med. 2017; 60(5): 494-503

45. Ahlbom A, Green A, Kheifets L, Savitz D, Swerdlow A. Epidemiology of Health Effects of Radiofrequency Exposure. Environ Health Perspect [Internet]. Diciembre de 2004 [citado 8 de enero de 2018]; 112(17):174154. Disponible en: https://www.ncbi.nlm.nih.gov/pmc/articles/PMC1253668/

46. Instituto Nacional de Seguridad e Higiene en el Trabajo. [Sede Web]. Madrid: INSHT; 2011. Berlana Llorente T, Diego Segura B, Rupérez Calvo MJ. Nota Técnica de Prevención nº 894: Campos electromagnéticos: evaluación de la exposición laboral. Disponible en: https://www.insst.es/ documents/94886/328579/894w.pdf/a2efcc9f-7491-4feb-8bb2-4ff72439019e

47. Stein Y, Levy Nativ O, Richter E. A sentinel case series of cancer patients with occupational exposures to electromagnetic non-ionizing radiation and other agents. Eur J Oncol. 2011; 16: 21-54.

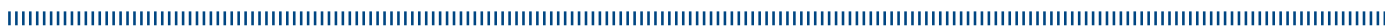




\section{ANEXOS}

Anexo I. Estudios que analizan la relación entre el tiempo de exposición a CEM y el riesgo de padecer cáncer de mama.

\begin{tabular}{|c|c|c|c|c|c|c|c|c|c|}
\hline Referencia & Año & Diseño & $\begin{array}{l}\text { Lugar y población } \\
\text { de estudio }\end{array}$ & Muestra & $\begin{array}{l}\text { Método utilizado } \\
\text { para medir } \\
\text { exposición }\end{array}$ & $\begin{array}{l}\text { Tiempo de } \\
\text { exposición }\end{array}$ & $\begin{array}{l}\text { Medida de } \\
\text { asociación }\end{array}$ & IC 95\% & NE* \\
\hline \multirow{2}{*}{$\begin{array}{l}\text { Van } \\
\text { Wijngaarden } \\
\text { et al. (16) }\end{array}$} & \multirow[t]{2}{*}{2001} & \multirow{2}{*}{$\begin{array}{l}\text { Casos y } \\
\text { controles }\end{array}$} & \multirow{2}{*}{$\begin{array}{c}\text { Mujeres de } 20-74 \\
\text { años con cáncer de } \\
\text { mama } 1993-1995 \text { del } \\
\text { Registro Central de } \\
\text { cáncer de Carolina } \\
\text { del Norte. }\end{array}$} & \multirow{2}{*}{$\begin{array}{c}843 \text { casos y } 773 \\
\text { controles }\end{array}$} & \multirow{2}{*}{$\begin{array}{l}\text {-Tabla de exposición } \\
\text { laboral } \\
\text {-indice de exposición } \\
\text { acumulada: promedio } \\
\text { ponderado en el } \\
\text { tiempo (TWA) }\end{array}$} & $\begin{array}{c}\text { Exposición total: } \\
0.59-0.90 \mu \mathrm{T} \text {-años } \\
0.90-1.27 \mu \mathrm{T} \text {-años } \\
1.27-2.43 \mu \mathrm{T} \text {-años } \\
>02.43 \mu \mathrm{T} \text {-años }\end{array}$ & $\begin{array}{l}O R=1.4 \\
O R=1.1 \\
O R=1.1 \\
O R=1.2\end{array}$ & $\begin{array}{l}1.1-1.8 \\
0.8-1.5 \\
0.8-1.4 \\
0.8-1.7\end{array}$ & \multirow[t]{2}{*}{$2-$} \\
\hline & & & & & & $\begin{array}{c}>20 \text { años: } \\
0-0.19 \mu \mathrm{T} \text {-años } \\
0.19-0.76 \mu \mathrm{T} \text {-años } \\
\geq 0.76 \mu \mathrm{T} \text {-años }\end{array}$ & $\begin{array}{l}\mathrm{OR}=1.4 \\
\mathrm{OR}=1.5 \\
\mathrm{OR}=1.2\end{array}$ & $\begin{array}{l}1.0-1.9 \\
1,1-2,0 \\
0.8-1.8\end{array}$ & \\
\hline \multirow{4}{*}{$\begin{array}{l}\text { Kliukiene et al. } \\
\text { (25) }\end{array}$} & \multirow{4}{*}{2003} & \multirow{4}{*}{$\begin{array}{l}\text { Casos y } \\
\text { controles } \\
\text { anidado }\end{array}$} & \multirow{4}{*}{$\begin{array}{l}\text { Mujeres noruegas } \\
\text { operadoras de radio } \\
\text { y teléfono }\end{array}$} & \multirow{4}{*}{$\begin{array}{l}99 \text { casos y } 396 \\
\text { controles }\end{array}$} & \multirow{4}{*}{$\begin{array}{c}\text { Categoria } \\
\text { exposición/años }\end{array}$} & Todas & $O R=1.43$ & $0.74-2.74$ & \multirow{4}{*}{$2+$} \\
\hline & & & & & & $0-<2,57 \mathrm{~Hz}$ /año & $\mathrm{OR}=1.27$ & $0.6-2.67$ & \\
\hline & & & & & & $2,57-<5,19 \mathrm{~Hz} /$ año & $O R=1.15$ & $0.54-2.47$ & \\
\hline & & & & & & $>5,19 \mathrm{~Hz} /$ año & $O R=2.01$ & $0.97-4.22$ & \\
\hline \multirow{4}{*}{$\begin{array}{l}\text { Johansen et } \\
\text { al. (34) }\end{array}$} & \multirow{4}{*}{2007} & \multirow{4}{*}{ Cohorte } & \multirow{4}{*}{$\begin{array}{c}\text { Empleadas de } \\
\text { empresas eléctricas } \\
\text { danesas } 1968-2002\end{array}$} & \multirow{4}{*}{$\begin{array}{c}28.224(22.436 \\
\text { hombres y } \\
5.788 \\
\text { mujeres) }\end{array}$} & \multirow{4}{*}{$\begin{array}{l}\text { Una matriz revisada } \\
\text { de exposición laboral }\end{array}$} & 6-9 años & $\mathrm{OR}=0.96$ & $0.89-1.02$ & \multirow{4}{*}{$2-$} \\
\hline & & & & & & $10-34$ años & $O R=1.00$ & $0.94-1.06$ & \\
\hline & & & & & & $35-40$ años & $\mathrm{OR}=0.98$ & $0.90-1.08$ & \\
\hline & & & & & & $\geq 41$ años & $\mathrm{OR}=0.98$ & $0.90-1.08$ & \\
\hline \multirow{3}{*}{$\begin{array}{l}\text { Labrèche et } \\
\text { al. (24) }\end{array}$} & \multirow{3}{*}{2003} & \multirow{3}{*}{$\begin{array}{l}\text { Casos y } \\
\text { controles }\end{array}$} & \multirow{3}{*}{$\begin{array}{c}\text { Trabajadoras } 50-75 \\
\text { años }\end{array}$} & \multirow{3}{*}{$\begin{array}{l}608 \text { casos y } 667 \\
\text { controles }\end{array}$} & \multirow{3}{*}{$\begin{array}{l}\text { Adjudicado por } \\
\text { higienistas según el } \\
\text { puesto de trabajo }\end{array}$} & toda la vida & $\mathrm{OR}=1.13$ & $0.94-1.35$ & \multirow{3}{*}{$2-$} \\
\hline & & & & & & $\begin{array}{c}10 \text { años antes del } \\
\text { diagnóstico }\end{array}$ & $O R=1.20$ & $0.98-1.48$ & \\
\hline & & & & & & $\begin{array}{l}\text { exposición antes } \\
\text { de los } 35 \text { años }\end{array}$ & $\mathrm{OR}=1.40$ & $0.98-2.02$ & \\
\hline
\end{tabular}

*NE: nivel de evidencia

Anexo II. Estudios que analizan la relación entre el tiempo de exposición a CEM y el riesgo de padecer algún tumor hematológico.

\begin{tabular}{|c|c|c|c|c|c|c|c|c|c|c|}
\hline Referencia & Añ̄o & Diseño & $\begin{array}{l}\text { Lugar y } \\
\text { población de } \\
\text { estudio }\end{array}$ & Muestra & $\begin{array}{l}\text { Tipo de } \\
\text { cáncer }\end{array}$ & $\begin{array}{l}\text { Método utilizado } \\
\text { para medir } \\
\text { exposición }\end{array}$ & $\begin{array}{l}\text { Tiempo de } \\
\text { exposición }\end{array}$ & Resultado & IC $95 \%$ & $\mathrm{NE}^{*}$ \\
\hline \multirow{2}{*}{$\begin{array}{l}\text { Fabro- } \\
\text { Peray et al. } \\
\text { (17) }\end{array}$} & \multirow{2}{*}{2001} & \multirow{2}{*}{$\begin{array}{l}\text { Casos y } \\
\text { controles }\end{array}$} & \multirow{2}{*}{$\begin{array}{c}\text { casos de } \\
\text { linforma } 1992 \text { - } \\
1995, \\
\text { controles de } \\
\text { listas } \\
\text { electorales }\end{array}$} & \multirow{2}{*}{$\begin{array}{c}445 \text { casos } \\
\text { y } 1963 \\
\text { controles }\end{array}$} & \multirow{2}{*}{$\begin{array}{l}\text { Linfoma } \\
\text { no } \\
\text { Hodgkin } \\
\text { (LNH) }\end{array}$} & \multirow{2}{*}{$\begin{array}{l}\text { Lista de trabajos } \\
\text { especificos y } \\
\text { frecuencia de } \\
\text { exposición } \\
\text { (ocasional, a } \\
\text { menudo, diaria) }\end{array}$} & $\begin{array}{c}\text { Radioperadores: } \\
\leq 9.5 \text { años } \\
>9.5 \text { años }\end{array}$ & $\begin{array}{l}O R=6.3 \\
O R=1.6\end{array}$ & $\begin{array}{l}2.1-18.9 \\
0.5-4.9\end{array}$ & \multirow{2}{*}{ 2- } \\
\hline & & & & & & & $\begin{array}{l}\text { Soldadores: } \\
\leq 13 \text { años } \\
>13 \text { años }\end{array}$ & $\begin{array}{l}\mathrm{OR}=2.7 \\
\mathrm{OR}=1.8\end{array}$ & $\begin{array}{l}1.3-5.5 \\
0.9-3.5\end{array}$ & \\
\hline $\begin{array}{l}\text { Tynes y } \\
\text { Haldorsen } \\
\text { (27) }\end{array}$ & 2003 & $\begin{array}{l}\text { Casos y } \\
\text { controles }\end{array}$ & $\begin{array}{l}\text { Residentes y } \\
\text { trabajadores } \\
\text { con leucemia }\end{array}$ & $\begin{array}{c}1068 \\
\text { casos y } \\
2126 \\
\text { controles }\end{array}$ & $\begin{array}{c}\text { Leucemia } \\
\text {, linfoma, } \\
\text { MM }\end{array}$ & $\begin{array}{l}\text { Método de Flynn } \\
\text { modificado }\end{array}$ & $\begin{array}{l}x \text { años }>31: \\
\text {-Leucemia } \\
\text {-Linfoma } \\
\text {-MM }\end{array}$ & $\begin{array}{l}O R=1.1 \\
O R=0.8 \\
O R=1.1\end{array}$ & $\begin{array}{l}0.7-1.6 \\
0.6-1.1 \\
0.7-1.8 \\
\end{array}$ & $2-$ \\
\hline \multirow{2}{*}{$\begin{array}{l}\text { Willet et al. } \\
\text { (26) }\end{array}$} & \multirow{2}{*}{2003} & \multirow{2}{*}{$\begin{array}{l}\text { Casos y } \\
\text { controles }\end{array}$} & \multirow{2}{*}{$\begin{array}{c}\text { Trabajadores y } \\
\text { población 16- } \\
69 \text { años }\end{array}$} & \multirow{2}{*}{$\begin{array}{c}764 \text { casos } \\
\text { y } 1510 \\
\text { controles }\end{array}$} & \multirow{2}{*}{$\begin{array}{l}\text { Leucemia } \\
\text { aguda }\end{array}$} & \multirow{2}{*}{$\begin{array}{l}\text { SOCy } \\
\text { clasificación } \\
\text { industrial } \\
\text { estándar }\end{array}$} & Actual & $\mathrm{OR}=0.9$ & $0.6-1.4$ & \multirow[b]{2}{*}{$2-$} \\
\hline & & & & & & & Pasada & $\mathrm{OR}=0.9$ & $0.6-1.2$ & \\
\hline \multirow{3}{*}{$\begin{array}{l}\text { Karipidis et } \\
\text { al. (37) }\end{array}$} & \multirow{3}{*}{2007} & \multirow{3}{*}{$\begin{array}{l}\text { Casos y } \\
\text { controles }\end{array}$} & \multirow{3}{*}{$\begin{array}{c}\text { Pacientes } \\
\text { entre } 20-74 \\
\text { años con } \\
\text { diagnóstico de } \\
\text { linfoma No } \\
\text { Hodgkin (LNH) } \\
\text { de } 2 \text { regiones } \\
\text { de Australia }\end{array}$} & \multirow{3}{*}{$\begin{array}{c}694 \text { casos } \\
\text { y } 694 \\
\text { controles }\end{array}$} & \multirow{3}{*}{$\begin{array}{c}\text { Linfoma } \\
\text { no } \\
\text { Hodgkin }\end{array}$} & \multirow{3}{*}{$\begin{array}{l}\text { Matriz de } \\
\text { exposición laboral } \\
\text { realizada en } \\
\text { EEUU }\end{array}$} & 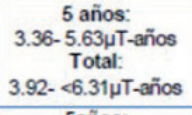 & $\begin{array}{l}\mathrm{OR}=0.94 \\
\mathrm{OR}=0.81\end{array}$ & $\begin{array}{l}0.67-1.32 \\
0.58-1.13 \\
\end{array}$ & \multirow{3}{*}{ 2- } \\
\hline & & & & & & & $\begin{array}{l}\text { 5años: } \\
\text { 5.63- } 9.11 \mu T \text {-años } \\
\text { Total: } \\
6.31-<9.85 \mu T \text {-años }\end{array}$ & $\begin{array}{l}O R=1.61 \\
O R=1.34\end{array}$ & $\begin{array}{l}1.12-2.33 \\
0.94-1.92\end{array}$ & \\
\hline & & & & & & & $\begin{array}{c}5 \text { anos: } \\
29.11 \mu \mathrm{T} \text {-años } \\
\text { Total: } \\
29.85 \mu \mathrm{T} \text {-años }\end{array}$ & $\begin{array}{l}O R=1.59 \\
O R=1.48\end{array}$ & $\begin{array}{l}1.07-2.36 \\
1.02-1.40\end{array}$ & \\
\hline
\end{tabular}

${ }^{*}$ NE: Nivel de evidencia 
Anexo III. Estudios que analizan la relación entre el tiempo de exposición a CEM y el riesgo de padecer algún tumor cerebral.

\begin{tabular}{|c|c|c|c|c|c|c|c|c|c|c|}
\hline Referencia & Año & Diseño & $\begin{array}{l}\text { Lugar y } \\
\text { población de } \\
\text { estudio }\end{array}$ & Muestra & $\begin{array}{l}\text { Tipo de } \\
\text { cáncer }\end{array}$ & $\begin{array}{l}\text { Método utilizado } \\
\text { para medir } \\
\text { exposición }\end{array}$ & $\begin{array}{l}\text { Tiempo de } \\
\text { exposición }\end{array}$ & $\begin{array}{l}\text { Medida de } \\
\text { asociación }\end{array}$ & IC $95 \%$ & $\mathrm{NE}^{*}$ \\
\hline \multirow{2}{*}{$\begin{array}{l}\text { Klaeboe et } \\
\text { al. (31) }\end{array}$} & \multirow{2}{*}{2005} & \multirow{2}{*}{$\begin{array}{l}\text { Casos y } \\
\text { controles } \\
\text { anidado }\end{array}$} & \multirow{2}{*}{$\begin{array}{c}\text { Población general } \\
>16 \text { años, } \\
\text { Noruega } 1980- \\
1996\end{array}$} & \multirow{2}{*}{$\begin{array}{l}454 \text { casos } 908 \\
\text { controles }\end{array}$} & \multirow{2}{*}{$\begin{array}{c}\text { Tumores } \\
\text { malignos, } \\
\text { glioma, } \\
\text { meningioma. }\end{array}$} & \multirow{2}{*}{$\begin{array}{l}\text { Modificación } \\
\text { método de Flynn }\end{array}$} & $\begin{array}{c}18-30 \\
\text { categoria/año } \\
\text {-todos tumores } \\
\text {-meningioma } \\
\text {-glioma }\end{array}$ & $\begin{array}{l}O R=0.8 \\
O R=0.3 \\
O R=1.1\end{array}$ & $\begin{array}{l}0.5-1.2 \\
0.1-0.9 \\
0.6-2.2\end{array}$ & \multirow[t]{2}{*}{$2-$} \\
\hline & & & & & & & $\begin{array}{c}231 \text { categoria/año } \\
\text {-todos tumores } \\
\text {-meningioma } \\
\text {-glioma }\end{array}$ & $\begin{array}{l}O R=0.6 \\
O R=0.1 \\
O R=0.8\end{array}$ & $\begin{array}{l}0.3-0.9 \\
0.0-0.5 \\
0.4-1.7\end{array}$ & \\
\hline \multirow{2}{*}{$\begin{array}{l}\text { Berg et al. } \\
\text { (36) }\end{array}$} & \multirow{2}{*}{2006} & \multirow{2}{*}{$\begin{array}{l}\text { casos y } \\
\text { controles }\end{array}$} & \multirow{2}{*}{$\begin{array}{l}\text { Población general } \\
\text { y casos en } 4 \\
\text { ciudades } \\
\text { alemanas entre } \\
2000-2003\end{array}$} & \multirow{2}{*}{$\begin{array}{c}366 \text { gliomas, } \\
381 \\
\text { meningiomas y } \\
1494 \text { contrdes }\end{array}$} & \multirow{2}{*}{$\begin{array}{l}\text { Glioma y } \\
\text { meningioma }\end{array}$} & \multirow{2}{*}{$\begin{array}{c}\text { Cuestionario } \\
\text { sobre trabajos } \\
\text { relacionados con } \\
\text { EMF (RF/MW- } \\
\text { EMF) }\end{array}$} & $\begin{array}{c}<10 \text { años } \\
\text {-Glioma } \\
\text {-Meningioma }\end{array}$ & $\begin{array}{l}O R=1.11 \\
O R=1.14\end{array}$ & $\begin{array}{l}0.48-2.56 \\
0.37-3.48\end{array}$ & \multirow{2}{*}{$2-$} \\
\hline & & & & & & & $\begin{array}{c}210 \text { años } \\
\text {-Glioma } \\
\text {-Meningioma }\end{array}$ & $\begin{array}{l}O R=1.39 \\
O R=1.55\end{array}$ & $\begin{array}{l}0.67-2.88 \\
0.52-4.62\end{array}$ & \\
\hline \multirow{3}{*}{$\begin{array}{l}\text { Karipidis et } \\
\text { al. (35) }\end{array}$} & \multirow{3}{*}{2007} & \multirow{3}{*}{$\begin{array}{l}\text { Casos y } \\
\text { controles }\end{array}$} & \multirow{3}{*}{$\begin{array}{c}\text { Pacientes entre } \\
20-70 \text { años con } \\
\text { diagnóstico de } \\
\text { glioma entre } \\
\text { 1987-1991, } \\
\text { Australia }\end{array}$} & \multirow{3}{*}{$\begin{array}{l}414 \text { casos y } 421 \\
\text { controles }\end{array}$} & \multirow{3}{*}{$\begin{array}{l}\text { glioma de } \\
\text { alto y bajo } \\
\text { grado }\end{array}$} & \multirow{3}{*}{$\begin{array}{c}\text { Auto-reportado, } \\
\text { higien ista experto } \\
\text { y JEM }\end{array}$} & $\begin{array}{c}\text { Autorreportado } \\
0-6 \text { años } \\
6-16 \text { años } \\
>16 \text { años }\end{array}$ & $\begin{array}{l}O R=0.85 \\
O R=0.83 \\
O R=0.57\end{array}$ & $\begin{array}{l}0.57-1.23 \\
0.57-1.21 \\
0.39-0.85\end{array}$ & \multirow{3}{*}{$2-$} \\
\hline & & & & & & & $\begin{array}{c}\text { Higienista experto } \\
0-4 \text { años } \\
4-13 \text { años } \\
>13 \text { años }\end{array}$ & $\begin{array}{l}O R=1.01 \\
O R=1.17 \\
O R=1.40\end{array}$ & $\begin{array}{l}0.04-0.00 \\
0.72-1.60 \\
0.85-2.27\end{array}$ & \\
\hline & & & & & & & 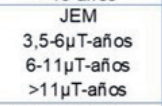 & $\begin{array}{l}O R=0.70 \\
O R=0.60 \\
O R=0.72\end{array}$ & $\begin{array}{l}0.47-1.04 \\
0,38-0.93 \\
0.44-1.19\end{array}$ & \\
\hline \multirow{3}{*}{$\begin{array}{l}\text { Karipidis et } \\
\text { al. (36) }\end{array}$} & \multirow{3}{*}{2007} & \multirow{3}{*}{$\begin{array}{l}\text { Casos y } \\
\text { controles }\end{array}$} & \multirow{3}{*}{$\begin{array}{l}\text { Casos con } \\
\text { diagnostico de } \\
\text { glioma entre } \\
1987-1991 \text { en } \\
\text { Australia }\end{array}$} & \multirow{3}{*}{$\begin{array}{c}416 \text { casos y } 422 \\
\text { controles }\end{array}$} & \multirow{3}{*}{ Glioma } & \multirow{3}{*}{$\begin{array}{l}\text { matriz de } \\
\text { exposición laboral } \\
\text { finlandesa } \\
\text { (FINJEM) }\end{array}$} & $0-0,75 \mu$ T-años & $\mathrm{OR}=0.76$ & $0.52-1.11$ & \multirow{3}{*}{$2-$} \\
\hline & & & & & & & $0,75-2,5 \mu \mathrm{T}$-años & $\mathrm{OR}=0.80$ & $0.55-1.17$ & \\
\hline & & & & & & & $>2,5 \mu \mathrm{T}$-años & $O R=0.79$ & $0.58-1.18$ & \\
\hline
\end{tabular}

*NE: Nivel de evidencia

Anexo III. Estudios que analizan la relación entre el tiempo de exposición a CEM y el riesgo de padecer algún tumor cerebral. (Continuación)

\begin{tabular}{|c|c|c|c|c|c|c|c|c|c|c|}
\hline Referencia & Año & Diseño & $\begin{array}{l}\text { Lugary } \\
\text { población de } \\
\text { estudio }\end{array}$ & Muestra & $\begin{array}{l}\text { Tipo de } \\
\text { cáncer }\end{array}$ & $\begin{array}{l}\text { Método utilizado } \\
\text { para medir } \\
\text { exposición }\end{array}$ & $\begin{array}{l}\text { Tiempo de } \\
\text { exposición }\end{array}$ & $\begin{array}{l}\text { Medida de } \\
\text { asociación }\end{array}$ & IC $95 \%$ & $\mathrm{NE}^{*}$ \\
\hline \multirow{4}{*}{ Coble et al. } & \multirow{4}{*}{2008} & \multirow{4}{*}{$\begin{array}{l}\text { Casos y } \\
\text { controles }\end{array}$} & \multirow{4}{*}{$\begin{array}{c}\text { Pacientes }>18 a \\
\text { en } 3 \text { hospitales de } \\
\text { EEUU. }\end{array}$} & \multirow{4}{*}{$\begin{array}{l}489 \text { caos de } \\
\text { glioma, } 197 \\
\text { casos de } \\
\text { meningioma y } \\
799 \text { controles }\end{array}$} & \multirow{4}{*}{$\begin{array}{c}\text { Glioma y } \\
\text { meningioma }\end{array}$} & \multirow{4}{*}{$\begin{array}{c}\text { Matriz de } \\
\text { exposición laboral } \\
\text { (JEM) }\end{array}$} & $\begin{array}{c}0-15 \text { años } \\
\text { Glioma } \\
\text { Meningioma }\end{array}$ & $\begin{array}{l}\mathrm{OR}=0.8 \\
\mathrm{OR}=1.0\end{array}$ & $\begin{array}{l}0.6-1.1 \\
0.7-1.6\end{array}$ & \multirow{4}{*}{$2-$} \\
\hline & & & & & & & $\begin{array}{c}>15 \text { años } \\
\text { Glioma } \\
\text { Meningioma }\end{array}$ & $\begin{array}{l}O R=0.8 \\
O R=1.0\end{array}$ & $\begin{array}{l}0.6-1.2 \\
0.6-1.7\end{array}$ & \\
\hline & & & & & & & $\begin{array}{l}\text { 0-45mG-años } \\
\text { Glioma } \\
\text { Meningioma }\end{array}$ & $\begin{array}{l}O R=0.8 \\
O R=10\end{array}$ & $\begin{array}{l}0.6-1.1 \\
0.7-16\end{array}$ & \\
\hline & & & & & & & $\begin{array}{l}>45 \mathrm{mG} \text {-años } \\
\text { Glioma } \\
\text { Meningioma }\end{array}$ & $\begin{array}{l}O R=0.8 \\
O R=1.0\end{array}$ & $\begin{array}{l}0.5-1.2 \\
0.6-1.8\end{array}$ & \\
\hline \multirow{4}{*}{$\begin{array}{l}\text { Carlberg et } \\
\text { al. }\end{array}$} & \multirow{4}{*}{2017} & \multirow{4}{*}{$\begin{array}{l}\text { Casos y } \\
\text { controles }\end{array}$} & \multirow{4}{*}{$\begin{array}{l}\text { Trabajadores 20- } \\
80 \text { años (1997-- } \\
2003) \text { y 18-75 } \\
\text { años }(2007-2009)\end{array}$} & \multirow{4}{*}{$\begin{array}{c}1346 \text { casos y } \\
3485 \text { controles }\end{array}$} & \multirow{4}{*}{$\begin{array}{c}\text { Glioma y } \\
\text { astrocitoma }\end{array}$} & \multirow{4}{*}{$\begin{array}{c}\text { Matriz de } \\
\text { exposición laboral } \\
\text { (INTEROCC ELF) }\end{array}$} & $\begin{array}{l}\text { 1.44-2.55u T-año } \\
\text { Glioma } \\
\text { Astrocitoma IV }\end{array}$ & $\begin{array}{l}\mathrm{OR}=0.8 \\
\mathrm{OR}=1.1\end{array}$ & $\begin{array}{l}0.7-0.9 \\
0.8-1.4\end{array}$ & \multirow{4}{*}{$2-$} \\
\hline & & & & & & & $\begin{array}{l}2.55-4.1 \mathrm{uT} \text {-año } \\
\text { Glioma } \\
\text { Astrocitoma IV }\end{array}$ & $\begin{array}{l}\mathrm{OR}=0.8 \\
\mathrm{OR}=1.0\end{array}$ & $\begin{array}{c}0.6-1.01 \\
0.7-1.3\end{array}$ & \\
\hline & & & & & & & $\begin{array}{l}\text { 4.17-6.59u T-año } \\
\text { Glioma } \\
\text { Astrocitoma IV }\end{array}$ & $\begin{array}{l}\mathrm{OR}=0.9 \\
\mathrm{OR}=0.9\end{array}$ & $\begin{array}{l}0.7-1.1 \\
0.7-1.3\end{array}$ & \\
\hline & & & & & & & $\begin{array}{l}\text { >6.59uT-año } \\
\text { Glioma } \\
\text { Astrocitoma IV }\end{array}$ & $\begin{array}{l}\mathrm{OR}=0.8 \\
\mathrm{OR}=0.9\end{array}$ & $\begin{array}{l}0.6-1.1 \\
0.6-13\end{array}$ & \\
\hline
\end{tabular}

${ }^{*}$ NE: nivel de evidencia 
Anexo IV. Estudios que analizan la relación entre los niveles de exposición a CEM y el riesgo de padecer algún tumor hematológico.

\begin{tabular}{|c|c|c|c|c|c|c|c|c|c|c|}
\hline Referencia & Año & Diseño & $\begin{array}{l}\text { Lugar y población } \\
\text { de estudio }\end{array}$ & Muestra & $\begin{array}{l}\text { Tipo de } \\
\text { cancer }\end{array}$ & $\begin{array}{l}\text { Método utilizado para } \\
\text { medir exposición }\end{array}$ & $\begin{array}{l}\text { Nivel de } \\
\text { exposición }\end{array}$ & $\begin{array}{l}\text { Medida de } \\
\text { asociación }\end{array}$ & IC $95 \%$ & NE* \\
\hline \multirow{2}{*}{$\begin{array}{l}\text { Villaneuve et al. } \\
\text { (13) }\end{array}$} & \multirow{2}{*}{2000} & \multirow{2}{*}{$\begin{array}{l}\text { Casos y con- } \\
\text { troles anida- } \\
\text { do }\end{array}$} & \multirow{2}{*}{$\begin{array}{c}\text { Empleados de em- } \\
\text { presa eléctrica y Re- } \\
\text { gistro de Cáncer On- } \\
\text { tario, Canada } 1970- \\
88\end{array}$} & \multirow{2}{*}{$\begin{array}{l}31543 \text { emplea- } \\
\text { dos } \\
\text { (Ratio casos: } \\
\text { controles 1:4) }\end{array}$} & \multirow{2}{*}{ Leucemia } & \multirow{2}{*}{$\begin{array}{l}\text { Monitor personal Positron } \\
\text { y JEM }\end{array}$} & $0.22-0.47 \mu \mathrm{T}$ & $O R=1.1$ & $0.28-4.28$ & \multirow{2}{*}{$2-$} \\
\hline & & & & & & & $>0.47 \mu T$ & $\mathrm{OR}=2.31$ & $0.66-8.12$ & \\
\hline \multirow{3}{*}{$\begin{array}{l}\text { Fabro-Peray et } \\
\text { al. (17) }\end{array}$} & \multirow{3}{*}{2001} & \multirow{3}{*}{$\begin{array}{l}\text { Casos y con- } \\
\text { troles }\end{array}$} & \multirow{3}{*}{$\begin{array}{l}\text { casos de linfoma } \\
\text { desde } 1992-1995, y \\
\text { controles de listas } \\
\text { electorales }\end{array}$} & \multirow{3}{*}{$\begin{array}{l}445 \text { casos } y \\
1963 \text { controles }\end{array}$} & \multirow{3}{*}{ LNH“* } & \multirow{3}{*}{$\begin{array}{l}\text { Lista de trabajos especiff- } \\
\text { cos y duración de exposi- } \\
\text { ción (expuesto> lafio) }\end{array}$} & $\begin{array}{l}2.1-5 \mathrm{mG} \\
\text { Pasado } \\
\text { Actual }\end{array}$ & $\begin{array}{l}O R=0.5 \\
O R=0.6\end{array}$ & $\begin{array}{l}0.1-2.4 \\
0.2-2.3\end{array}$ & \multirow{3}{*}{2 - } \\
\hline & & & & & & & $\begin{array}{l}\text { 5-10mG } \\
\text { Pasado } \\
\text { Actual }\end{array}$ & $\begin{array}{l}O R=2.9 \\
O R=1.5\end{array}$ & $\begin{array}{l}0.7-11.4 \\
0.2-14.6\end{array}$ & \\
\hline & & & & & & & $\begin{array}{l}>10 \mathrm{mG} \\
\text { Pasado } \\
\text { Actual }\end{array}$ & $\begin{array}{l}O R=3.2 \\
O R=4.0\end{array}$ & $\begin{array}{l}1.2-8.3 \\
1.6-9.8\end{array}$ & \\
\hline \multirow{7}{*}{$\begin{array}{l}\text { Betwhaite et al. } \\
\text { (18) }\end{array}$} & \multirow{7}{*}{2001} & \multirow{7}{*}{$\begin{array}{l}\text { Casos y con- } \\
\text { troles }\end{array}$} & \multirow{7}{*}{$\begin{array}{l}\text { Casos en N.Zelanda } \\
1989-1991 \text {. } \\
\text { Control: listas electo- } \\
\text { rales }\end{array}$} & \multirow{7}{*}{$\begin{array}{c}110 \text { casos y } 199 \\
\text { controles }\end{array}$} & \multirow{7}{*}{$\begin{array}{l}\text { LAL"*y } \\
\text { LANL" }\end{array}$} & \multirow{7}{*}{$\begin{array}{l}\text { JEM y datos de estudio } \\
\text { previos (Bowman 1992) }\end{array}$} & $\begin{array}{l}\text { Ingeniero } \\
\text { Eléctrico }\end{array}$ & $O R=0.4$ & $0.1-4.3$ & \multirow{7}{*}{$2-$} \\
\hline & & & & & & & Electricista & $O R=0.7$ & $0.3-1.3$ & \\
\hline & & & & & & & $\begin{array}{l}\text { Conductor } \\
\text { de tren }\end{array}$ & $O R=2.6$ & $0.7-9.3$ & \\
\hline & & & & & & & $\begin{array}{l}\text { Radio- } \\
\text { operador }\end{array}$ & $O R=3.3$ & $1.5-7.1$ & \\
\hline & & & & & & & $\begin{array}{c}\text { Operador de } \\
\text { Radar }\end{array}$ & $O R=1.3$ & $0.5-3.3$ & \\
\hline & & & & & & & Telegrafista & $O R=1.3$ & $0.5-3.3$ & \\
\hline & & & & & & & Soldador & $O R=2.6$ & $1.4-5.1$ & \\
\hline \multirow{2}{*}{$\begin{array}{l}\text { Johansen et al. } \\
\qquad(34)\end{array}$} & \multirow{2}{*}{2007} & \multirow{2}{*}{ Cohorte } & \multirow{2}{*}{$\begin{array}{l}\text { Empleados de em- } \\
\text { presas electricas da- } \\
\text { nesas, desde el } 1968 \\
-2002\end{array}$} & \multirow{2}{*}{$\begin{array}{c}28.224 \text { (22.438 } \\
\text { hombres y } 5.788 \\
\text { mujeres) }\end{array}$} & \multirow{2}{*}{ Leucemia } & \multirow{2}{*}{$\begin{array}{l}\text { Matriz revisada de exposi- } \\
\text { ción laboral }\end{array}$} & $0,1-0,99 \mu \mathrm{T}$ & $O R=0.97$ & $0.51-1.85$ & \multirow{2}{*}{2.} \\
\hline & & & & & & & $>1.0 \mu T$ & $O R=1.04$ & $0.53-2.04$ & \\
\hline $\begin{array}{l}\text { Koeman et al. } \\
\text { (35) }\end{array}$ & 2014 & $\begin{array}{c}\text { Cohorte } \\
\text { prospectivo }\end{array}$ & $\begin{array}{c}\text { Paises Bajos, hom- } \\
\text { bres y } \\
\text { mujeres en } 1986 \\
\text { (durante } 17.3 \text { años) }\end{array}$ & $\begin{array}{c}120.852 \text { sujetos } \\
\text { (58.279 hom- } \\
\text { bres y } \\
62.573 \text { mujeres) }\end{array}$ & $\begin{array}{l}\text { LFF" } \\
\text { LMA }\end{array}$ & $\begin{array}{c}\text { Matriz de exposición labo- } \\
\text { ral desarrollada reciente- } \\
\text { mente }\end{array}$ & $\begin{array}{l}\text { Exposición } \\
\text { elevada: } \\
\text { LMA } \\
\text { LF }\end{array}$ & $\begin{array}{l}H R=2,15 \\
H R=2.78\end{array}$ & $\begin{array}{l}1.06-4.35 \\
1.00-5.77\end{array}$ & 2. \\
\hline
\end{tabular}

${ }^{*}$ NE: nivel de evidencia. * ${ }^{*}$ Ver glosario 
Anexo V. Estudios que analizan la relación entre los niveles de exposición a CEM y el riesgo de padecer cáncer de mama.

\begin{tabular}{|c|c|c|c|c|c|c|c|c|c|c|c|c|c|c|c|c|c|}
\hline$\dot{\grave{z}}$ & \multicolumn{3}{|c|}{$\dot{\sim}$} & \multicolumn{3}{|c|}{ \pm} & \multicolumn{2}{|c|}{$\dot{\sim}$} & \multicolumn{2}{|r|}{$\dot{\sim}$} & \multicolumn{2}{|r|}{$\dot{\sim}$} & $\therefore$ & \multicolumn{2}{|c|}{$\dot{\sim}$} & \multicolumn{2}{|l|}{$\dot{\sim}$} \\
\hline $\begin{array}{l}\text { 总 } \\
\underline{0} \\
\underline{0}\end{array}$ & $\stackrel{0}{\grave{1}}$ & : & के & 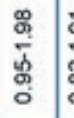 & 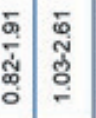 & 通 & 导 & 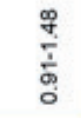 & 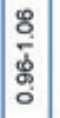 & $\begin{array}{l}8 \\
\vdots \\
\vdots \\
\vdots \\
\vdots\end{array}$ & 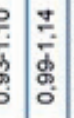 & 商 & 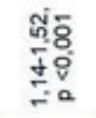 & $\begin{array}{l}\stackrel{5}{-} \\
\dot{0} \\
\stackrel{0}{0} \\
0\end{array}$ & $\begin{array}{l}\text { लू } \\
\text { ల్లై } \\
\text { है }\end{array}$ & 胥 & 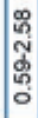 \\
\hline 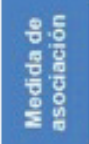 & $\begin{array}{l}\bar{\pi} \\
\frac{\pi}{\alpha}\end{array}$ & $\mid$\begin{tabular}{|l}
$\bar{\pi}$ \\
$\frac{\pi}{\alpha}$
\end{tabular} & $\underset{\substack{\alpha \\
\alpha}}{\bar{\alpha}}$ & $\frac{\hat{m}}{\bar{c}}$ & 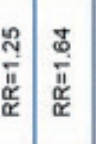 & $\begin{array}{l}\tilde{\delta} \\
\delta_{0} \\
\alpha \\
\alpha \\
\alpha\end{array}$ & $\frac{m}{\bar{a}}$ & 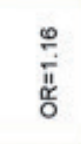 & 产 & 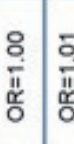 & 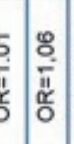 & 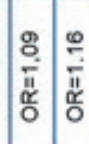 & 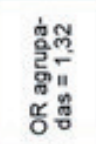 & $\begin{array}{l}\text { R. } \\
\text { ô }\end{array}$ & ti & $\frac{\hat{t}}{i 1}$ & $\frac{\pi}{\pi}$ \\
\hline 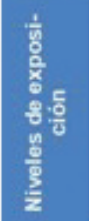 & 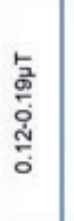 & 詹 & 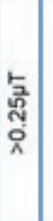 & 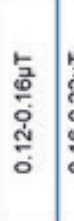 & 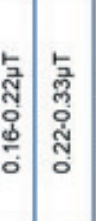 & 氛 & 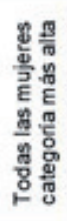 & 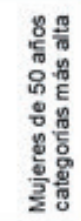 & 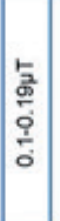 & 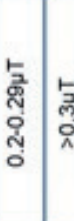 & 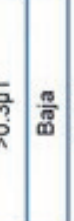 & $\frac{\frac{\pi}{8}}{\frac{8}{2}} \frac{\frac{\pi}{\alpha}}{\alpha}$ & 1 & 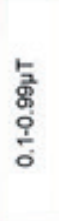 & $\stackrel{\text { 홍 }}{-}$ & $\frac{m}{\bar{m}}$ & $\frac{m}{4}$ \\
\hline $\begin{array}{l}8 \\
\frac{1}{8} \\
8 \\
8\end{array}$ & 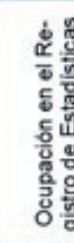 & 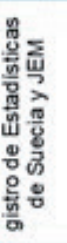 & & & 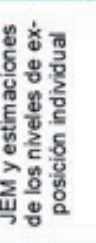 & & & & & 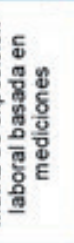 & & 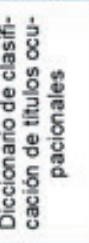 & 1 & & & 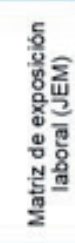 & \\
\hline 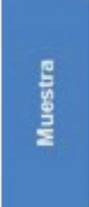 & 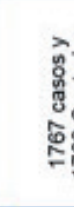 & 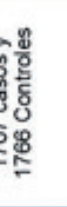 & & & 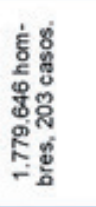 & & & & & 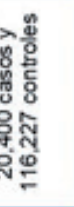 & & 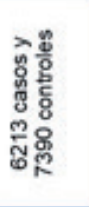 & 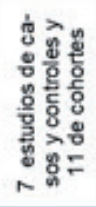 & & & 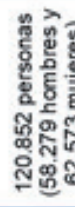 & \\
\hline 曾 & 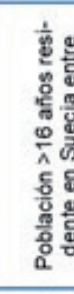 & 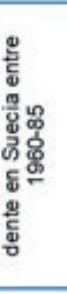 & & & 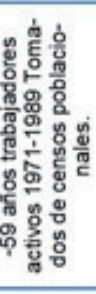 & & & 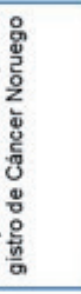 & & 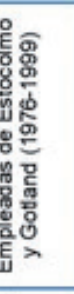 & & 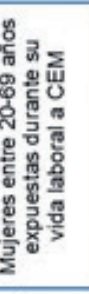 & 1 & & 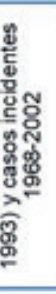 & 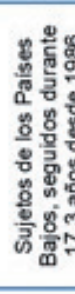 & \\
\hline o & 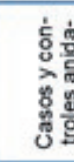 & 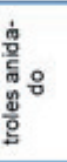 & & & $\begin{array}{l}\text { \%ั้ } \\
\text { ㅎํㅇ }\end{array}$ & & & & & 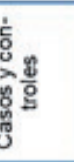 & & 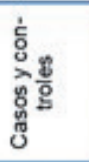 & 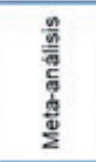 & & & 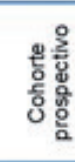 & \\
\hline 造 & & ర్ల & & & $\bar{\delta}$ & & & & & ర్ల & & ఫ్రి & స్ & & & సั & \\
\hline & & 势 & & & 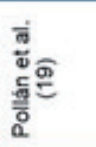 & & & & & 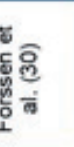 & & 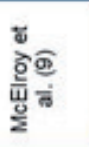 & 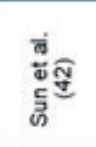 & & & 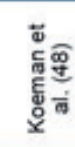 & \\
\hline
\end{tabular}

*NE: nivel de evidencia 
Anexo VI. Estudios que analizan la relación entre los niveles de exposición a CEM y el riesgo de padecer algún tumor cerebral.

\begin{tabular}{|c|c|c|c|c|c|c|c|c|c|c|c|c|c|c|c|c|}
\hline & $\dot{N}$ & \multicolumn{2}{|c|}{$\dot{\Lambda}$} & \multicolumn{3}{|c|}{$\dot{\sim}$} & \multicolumn{2}{|l|}{$\dot{\alpha}$} & \multicolumn{2}{|c|}{$\dot{\lambda}$} & $\dot{\Lambda}$ & \multicolumn{2}{|c|}{$\dot{\alpha}$} & \multicolumn{3}{|c|}{$\dot{\alpha}$} \\
\hline & 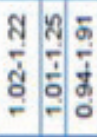 & 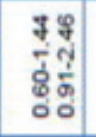 & 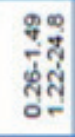 & $\begin{array}{l}\frac{q}{2} \\
\frac{\$}{\phi} \\
0 \\
0\end{array}$ & 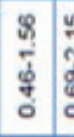 & $\begin{array}{c}5 \\
7 \\
\$ \\
\%\end{array}$ & $\begin{array}{c}50 \\
\frac{5}{5} \\
\frac{5}{5} \\
0\end{array}$ & क्ष & 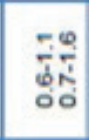 & 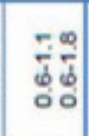 & 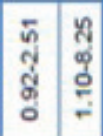 & 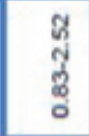 & हू & केष & 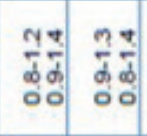 & : \\
\hline & $\frac{N}{\alpha} \frac{\substack{\alpha \\
\alpha}}{\frac{\alpha}{\alpha}}$ & 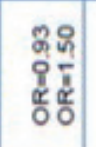 & 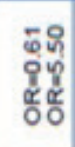 & $\begin{array}{l}\text { \$ } \\
0 \\
0 \\
0 \\
0\end{array}$ & 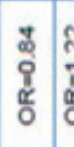 & สุ| & 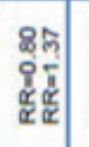 & 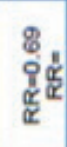 & 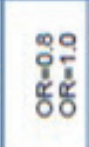 & 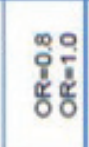 & 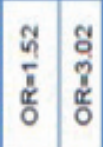 & $\frac{\vartheta}{\frac{\alpha}{x}}$ & $\begin{array}{l}k \\
0 \\
0 \\
\frac{d}{x}\end{array}$ & ํํㄹำ & 움 & \begin{tabular}{ll}
0 \\
\hdashline \\
\hdashline \\
$\dot{x}$
\end{tabular} \\
\hline & 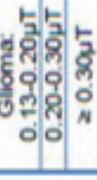 & 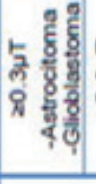 & 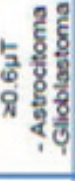 & $\begin{array}{l}\frac{2}{2} \\
\frac{1}{3} \\
\frac{2}{2} \\
\frac{9}{2} \\
\end{array}$ & $\begin{array}{l}\frac{2}{3} \\
\frac{8}{8} \\
\frac{8}{2}\end{array}$ & $\frac{5}{2}$ & 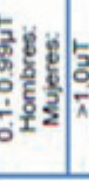 & 6. & 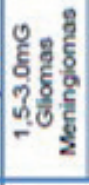 & 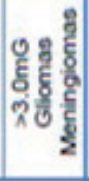 & 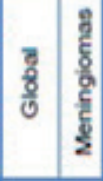 & $\frac{8}{8}$ & $\frac{2}{8}$ & 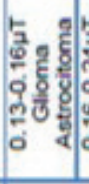 & 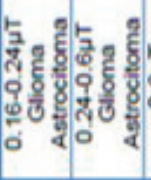 & 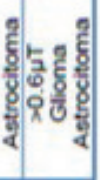 \\
\hline & 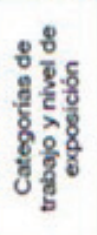 & 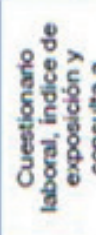 & 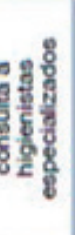 & & 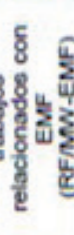 & & 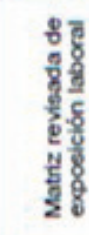 & & $\begin{array}{l}8 \\
8 \\
\frac{8}{8} \\
\frac{5}{2} \\
\frac{8}{2} \\
\frac{8}{2}\end{array}$ & s. & 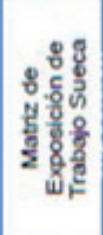 & & & & 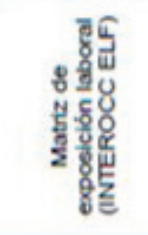 & \\
\hline & 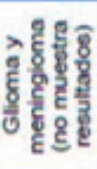 & 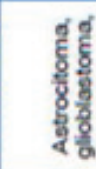 & & & & & $>$ 象 & & $\frac{8}{0}$ & 优 & 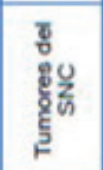 & & & & 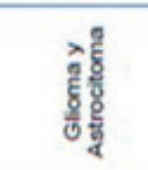 & \\
\hline & 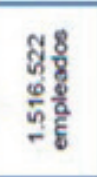 & $\begin{array}{l}\text { रे. } \\
\text { s. } \\
\text { है? }\end{array}$ & & & 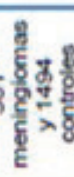 & & 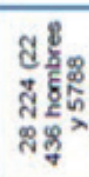 & & 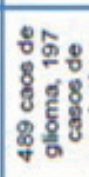 & 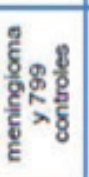 & 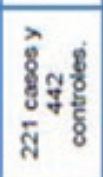 & 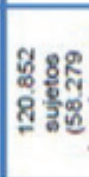 & & & 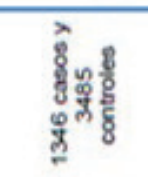 & \\
\hline & 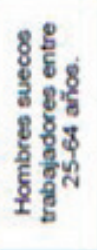 & 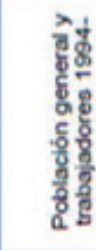 & & & 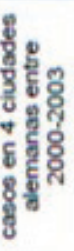 & & 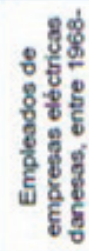 & & 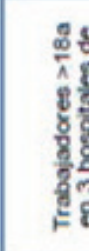 & خ & 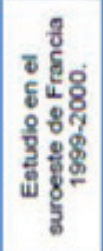 & 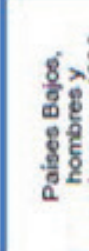 & & & 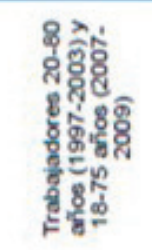 & \\
\hline & ㅇํㅇ & $\begin{array}{l}\overrightarrow{8} \\
\delta \\
\delta\end{array}$ & & & 8 & & ㅇํㅇ & & 8 & & $\begin{array}{l}>8 \\
8 \frac{8}{5} \\
88^{2}\end{array}$ & & & & 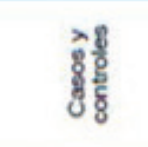 & \\
\hline & ర్ల & ¿্ & & & 8 & & हे & & $\varnothing$ & & $\bar{\alpha}$ & 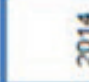 & & & $\hat{\bar{\alpha}}$ & \\
\hline & 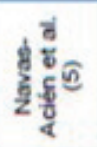 & క్ & & & 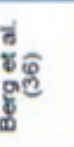 & & 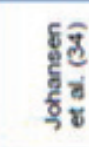 & & ฮู & 8 & हैं & & $\widehat{\vartheta}$ & & 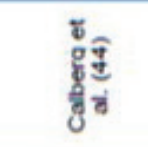 & \\
\hline
\end{tabular}

${ }^{*}$ NE: nivel de evidencia 
Anexo VII. Estudios que analizan la relación entre exposición ocupacional a CEM y otros cánceres.

\begin{tabular}{|c|c|c|c|c|c|c|c|c|c|}
\hline Referencia & Año & Diseño & $\begin{array}{l}\text { Lugar y población } \\
\text { de estudio }\end{array}$ & Muestra & $\begin{array}{l}\text { Método utilizado } \\
\text { para medir } \\
\text { exposición }\end{array}$ & $\begin{array}{l}\text { Tipo de } \\
\text { cáncer }\end{array}$ & $\begin{array}{l}\text { Categoria de } \\
\text { exposición }\end{array}$ & $\begin{array}{l}\text { Resultados } \\
\text { (medida e } \\
\text { IC95\%) }\end{array}$ & NE* \\
\hline$\frac{\text { Fincham et }}{\text { al. (15) }}$ & 2000 & $\begin{array}{c}\text { Casos y } \\
\text { controles }\end{array}$ & $\begin{array}{l}\text { Canadá, Registros } \\
\text { de Cáncer, del } \\
\text { hospital y bases de } \\
\text { datos de la } \\
\text { administración } \\
\text { provincial. }\end{array}$ & $\begin{array}{l}1272 \text { casos } \\
\text { y } 2666 \\
\text { controles }\end{array}$ & $\begin{array}{c}\text { Clasificación de } \\
\text { historia laboral de } \\
\text { acuerdo a la posible } \\
\text { exposición a CEM }\end{array}$ & $\begin{array}{l}\text { Cáncer de } \\
\text { tiroides }\end{array}$ & $\begin{array}{l}\text { Categoria } \\
\text { ocupacional }\end{array}$ & $\begin{array}{c}O R=1.59 \\
(0.80-3.15)\end{array}$ & 2. \\
\hline \multirow{2}{*}{$\begin{array}{l}\text { Stang et al. } \\
\text { (20) }\end{array}$} & \multirow{2}{*}{2001} & \multirow{2}{*}{$\begin{array}{c}\text { casos y } \\
\text { controles }\end{array}$} & \multirow{2}{*}{ Alemania. } & \multirow{2}{*}{$\begin{array}{c}118 \text { casos y } \\
475 \\
\text { controles }\end{array}$} & \multirow{2}{*}{$\begin{array}{l}\text { Encuesta que } \\
\text { incluye la exposición } \\
\text { ocupacional }\end{array}$} & \multirow{2}{*}{$\begin{array}{l}\text { Melanoma } \\
\text { uveal }\end{array}$} & \multirow{2}{*}{$\begin{array}{l}\text { Categoria } \\
\text { ocupacional }\end{array}$} & $\begin{array}{l}\text { máquinas } \\
\text { eléctricas } \\
\mathrm{OR}=1.0 \\
(0.6-1.7)\end{array}$ & \multirow{2}{*}{2 - } \\
\hline & & & & & & & & $\begin{array}{c}\text { pantallas de } \\
\text { visualización } \\
\text { OR=1.1 } \\
(0.6-1.9) \\
\end{array}$ & \\
\hline $\begin{array}{l}\text { Baumgardt- } \\
\text { Elms et al. } \\
\text { (22) }\end{array}$ & 2002 & $\begin{array}{l}\text { Casos y } \\
\text { controles }\end{array}$ & $\begin{array}{c}\text { Trabajadores y } \\
\text { población general } \\
15-69 \text { años, } \\
\text { Alemania }\end{array}$ & $\begin{array}{c}269 \text { casos y } \\
797 \\
\text { controles }\end{array}$ & $\begin{array}{l}5 \text { categorias de } \\
\text { exposicion }(A-E)\end{array}$ & Testicular & $\begin{array}{l}\text { Categoria } \\
\text { EMF }\end{array}$ & $\begin{array}{l}\text { Categoria A: } \\
\text { OR=0.9 } \\
(0.67-1.21)\end{array}$ & $2-$ \\
\hline \multirow{2}{*}{$\begin{array}{l}\text { Tynes et al. } \\
\text { (28) }\end{array}$} & \multirow[b]{2}{*}{2003} & \multirow{2}{*}{$\begin{array}{l}\text { casos y } \\
\text { controles } \\
\text { anidado }\end{array}$} & \multirow{2}{*}{$\begin{array}{c}\text { Trabajadores y } \\
\text { población general } \\
>16 \text { años }\end{array}$} & \multirow{2}{*}{$\begin{array}{c}807 \text { casos y } \\
1614 \\
\text { controles }\end{array}$} & \multirow{2}{*}{$\begin{array}{l}\text { Método de Flynn } \\
\text { modificado }\end{array}$} & \multirow[b]{2}{*}{ Melanoma } & \multirow{2}{*}{$\begin{array}{l}\text { Categoria } x \\
\text { años }\end{array}$} & $\begin{array}{l}\text { 18-30 años: } \\
\text { OR=1.05 } \\
(0.8-1.45)\end{array}$ & \multirow[b]{2}{*}{$2-$} \\
\hline & & & & & & & & $\begin{array}{l}>30 \text { años: } \\
\text { OR=1.22 } \\
(0.81-1.82)\end{array}$ & \\
\hline \multirow{2}{*}{$\begin{array}{l}\text { Lope et al. } \\
\text { (33) }\end{array}$} & \multirow{2}{*}{2006} & \multirow{2}{*}{$\begin{array}{l}\text { Cohorte } \\
\text { histórico }\end{array}$} & \multirow{2}{*}{$\begin{array}{c}\text { Sujetos de Suecia } \\
\text { seguidos entre } \\
\text { 1971-1989 del censo } \\
\text { de } 1970 .\end{array}$} & \multirow{2}{*}{$\begin{array}{c}2.992 .166 \\
\text { trabajadores } \\
y \\
\text { trabajadoras }\end{array}$} & \multirow{2}{*}{$\begin{array}{l}\text { Matriz de ocupación } \\
\text { laboral (JEM) }\end{array}$} & \multirow[t]{2}{*}{ Tiroides } & \multirow{2}{*}{$\begin{array}{c}\text { ALta } \\
\text { exposición }\end{array}$} & $\begin{array}{l}\text { Mujeres: } \\
\text { RR=1.85 } \\
(1.02-3.35)\end{array}$ & \multirow[t]{2}{*}{$2-$} \\
\hline & & & & & & & & $\begin{array}{l}\text { Hombres: } \\
\text { No medido }\end{array}$ & \\
\hline $\begin{array}{l}\text { Behrens et } \\
\text { al. (40) }\end{array}$ & 2010 & $\begin{array}{l}\text { Casos y } \\
\text { controles }\end{array}$ & $\begin{array}{c}\text { Pacientes } \\
\text { diagnosticados entre } \\
1994 \text { y } 1997 \text { en } 9 \\
\text { paises europeos }\end{array}$ & $\begin{array}{c}293 \text { casos y } \\
3198 \\
\text { controles }\end{array}$ & $\begin{array}{c}\text { Fómula que valora } \\
\text { exposición } \\
\text { acumulada por años } \\
\text { de exposición }\end{array}$ & $\begin{array}{c}\text { Melanoma } \\
\text { uveal }\end{array}$ & $\begin{array}{l}\text { Exposición } \\
\text { acumulada } \\
\text { por años de } \\
\text { exposición }\end{array}$ & $\begin{array}{c}\text { mujeres: } \\
\text { OR=5.81 } \\
(1.72- \\
19.66)\end{array}$ & 2 \\
\hline $\begin{array}{l}\text { Koeman et } \\
\text { al. (43) }\end{array}$ & 2014 & $\begin{array}{l}\text { Cohorte } \\
\text { prospectivo }\end{array}$ & $\begin{array}{c}\text { Sujetos de los } \\
\text { Paises Bajos, } \\
\text { seguidos } 17.3 \text { años } \\
\text { desde } 1986\end{array}$ & $\begin{array}{c}120.852 \\
\text { personas } \\
(58.279 \\
\text { hombres y } \\
62.573 \\
\text { mujeres })\end{array}$ & $\begin{array}{c}\text { Matriz de exposición } \\
\text { laboral }\end{array}$ & $\begin{array}{l}\text { Cáncer de } \\
\text { pulmón }\end{array}$ & $\begin{array}{l}\text { Categorias } \\
\text { por } \\
\text { intensidad }\end{array}$ & $\begin{array}{l}\text {-Hombres: } \\
\text { HR=1.29 } \\
(0.99-1.70) \text { - } \\
\text { Mujeres: } \\
\text { HR=1.23 } \\
(0.37-4.07)\end{array}$ & 2 - \\
\hline
\end{tabular}

*NE: nivel de evidencia 